\title{
OPTIMAL SOBOLEV REGULARITY FOR THE STOKES EQUATIONS ON A 2D WEDGE DOMAIN
}

\author{
MATTHIAS KÖHNE, JÜRGEN SAAL, AND LAURA WESTERMANN
}

\begin{abstract}
In this note we prove that the solution of the stationary and the instationary Stokes equations subject to perfect slip boundary conditions on a $2 \mathrm{D}$ wedge domain admits optimal regularity in the $L^{p}$-setting, in particular it is $W^{2, p}$ in space. This improves known results in the literature to a large extend. For instance, in 21, Theorem 1.1 and Corollary 3] it is proved that the Laplace and the Stokes operator in the underlying setting have maximal regularity. In that result the range of $p$ admitting $W^{2, p}$ regularity, however, is restricted to the interval $1<p<1+\delta$ for small $\delta>0$, depending on the opening angle of the wedge. This note gives a detailed answer to the question, whether the optimal Sobolev regularity extends to the full range $1<p<\infty$. We will show that for the Laplacian this does only hold on a suitable subspace, but, depending on the opening angle of the wedge domain, not for every $p \in(1, \infty)$ on the entire $L^{p}$-space. On the other hand, for the Stokes operator in the space of solenoidal fields $L_{\sigma}^{p}$ we obtain optimal Sobolev regularity for the full range $1<p<\infty$ and for all opening angles less than $\pi$. Roughly speaking, this relies on the fact that an existing "bad" part of $L^{p}$ for the Laplacian is complemented to the space of solenoidal vector fields.
\end{abstract}

\section{INTRODUCTION AND MAIN RESUlts}

It is well-known that regularity properties for PDE on non-smooth domains are important for many applications. The main objective of this note is to derive best possible regularity in the $L^{p}$-setting for the instationary Stokes equations

$$
\begin{aligned}
& \partial_{t} u-\Delta u+\nabla \pi=f \quad \text { in } \quad(0, \infty) \times G, \\
& \operatorname{div} u=0 \quad \text { in } \quad(0, \infty) \times G, \\
& \left.\begin{array}{rl}
\operatorname{curl} u=0, u \cdot \nu=0 & \text { on } \quad(0, \infty) \times \partial G, \\
u(0)=u_{0} & \text { in } \quad G,
\end{array}\right\}
\end{aligned}
$$

subject to perfect slip boundary conditions on a two-dimensional wedge type domain given as

$$
G:=\left\{\left(x_{1}, x_{2}\right) \in \mathbb{R}^{2}: 0<x_{2}<x_{1} \tan \theta_{0}\right\} .
$$

Here $\nu$ denotes the outer normal vector at $\partial G, \theta_{0} \in(0, \pi)$ the opening angle of the wedge, and $\operatorname{curl} u=\partial_{1} u_{2}-\partial_{2} u_{1}$.

There exist approaches to an $L^{p}$-theory for classical elliptic and parabolic problems on domains with conical boundary points, see, e.g., the classical monographs [10, 22], or also [3, 4] for the heat equation subject to Dirichlet boudary conditions. In contrast to that, corresponding results for the Stokes equations are very rare, in particular for the instationary case. For the stationary Stokes equations there are the classical regularity results [18, 17, 5, 22, 10, 7. For a negative result concerning the generation of an analytic semigroup in three dimensions for the Stokes operator subject to the no-slip

2010 Mathematics Subject Classification. Primary: 35Q30, 76D03, 35K67; Secondary: 76D05, 35K65.

Key words and phrases. Stokes equations, Laplace equation, wedge domains, perfect slip, optimal regularity. 
condition see [8]. More recently, an approach to analytic regularity was presented in [11]. We also refer to [19], where the Stokes equations subject to no-slip boundary conditions in a cone are studied, and to [13] for an overview on the Stokes equations including approaches to non-smooth domains.

It seems that a general approach to the instationary Stokes equations on domains with edges and vertices does not exist in the literature, even for domains having a simple structure such as wedge domains. There is, of course, the Lipschitz approach to even more general non-smooth domains. Existence and analyticity of the Stokes semigroup on $L_{\sigma}^{p}$ on Lipschitz domains is proved, for instance, in [23, 28, 30. Note that the Lipschitz approach does not provide full $W^{2, p}$ Sobolev regularity which, however, might be crucial for the treatment of related quasilinear problems. Moreover, in the Lipschitz approach the range of available $p$ is restricted in general. Thus, for our purposes this approach seems to be too general. The main objective of this note is $W^{2, p}$ Sobolev regularity for (1.1) for all $p \in(1, \infty)$.

Remark 1.1. In this note we frequently use the expressions maximal regularity, optimal (Sobolev) regularity, optimal regularity in the $L^{p}$-setting. The maximal regularity here refers to the standard maximal regularity concerning (linear) evolution equations, see [16, 6, 20, 27] for a precise definition. The latter two notions refer to optimal regularity in the sense of elliptic regularity, i.e., for the second order equations considered here to $W^{2, p}$-regularity in space.

Concerning Stokes the advantage of imposing perfect slip conditions lies in the fact that Helmholtz projector and Laplacian commute, which is not the case in general. Hence the Stokes operator is given as the part of the Laplacian in the solenoidal subspace. Note that this observation has been utilized in [23] and 21] already. In fact, in [21] maximal regularity for (1.1) is proved in two and three dimensional wedges in Kondrat'ev spaces

$$
L_{\gamma}^{p}\left(G, \mathbb{R}^{2}\right):=L^{p}\left(G, \rho^{\gamma} d\left(x_{1}, x_{2}\right), \mathbb{R}^{2}\right), \quad \rho:=\left|\left(x_{1}, x_{2}\right)\right|, \gamma \in \mathbb{R} .
$$

(Note that 21] focuses on the 3D version; the 2D counterpart then is completely analogous.) Optimal Sobolev regularity in the sense of our main results below, however, could only be established for $1<p<1+\delta$ with $\delta>0$ possibly small, depending on the opening angle $\theta_{0}$ of the wedge and the Kondrat'ev exponent $\gamma$. This shortcoming relies on a spectral constraint that relates to the constraint (1.6) in Theorem 1.4 below. In fact, for $\gamma=0$ under the constraint imposed in [21] we even have $\delta \rightarrow 0$ for $\theta_{0} \rightarrow \pi$ such that for angles close to $\pi$ only a very small interval for $p$ remains. Note that a similar spectral constraint concerning regularity for the heat equation subject to Dirichlet and Neumann boundary conditions is imposed in [25, 26.

In this note we will show that in $2 \mathrm{D}$ this vast restriction on $p$ can be dropped completely. To be precise, our main result reads as follows (see (3.1) and (3.2) for the definition of the solenoidal subspace $L_{\sigma}^{p}(G)$ on a wedge domain and the definition of $\left.\widehat{W}^{1, p}(G)\right)$.

Theorem 1.2. Let $1<p<\infty, \theta_{0} \in(0, \pi), \rho=\left|\left(x_{1}, x_{2}\right)\right|$, and $G \subset \mathbb{R}^{2}$ be defined as in (1.2). Then the Stokes operator subject to perfect slip

$$
\begin{aligned}
A_{S} u= & -\Delta u, \\
u \in D\left(A_{S}\right)= & \left\{u \in W^{2, p}\left(G, \mathbb{R}^{2}\right) \cap L_{\sigma}^{p}(G): \operatorname{curl} u=0, \nu \cdot u=0 \text { on } \partial G,\right. \\
& \left.\rho^{|\alpha|-2} \partial^{\alpha} u \in L^{p}\left(G, \mathbb{R}^{2}\right)(|\alpha| \leq 2)\right\}
\end{aligned}
$$

is $\mathcal{R}$-sectorial with $\mathcal{R}$-angle $\phi_{A_{S}}^{\mathcal{R}}<\pi / 2$, hence has maximal regularity on $L_{\sigma}^{p}(G)$. 
As an immediate consequence we obtain strong solvability of (1.1).

Corollary 1.3. Let $1<p, q<\infty, \theta_{0} \in(0, \pi), \rho=\left|\left(x_{1}, x_{2}\right)\right|$, and $G \subset \mathbb{R}^{2}$ be defined as in (1.2). Then for every $f \in L^{q}\left((0, \infty), L_{\sigma}^{p}(G)\right)$ and $u_{0} \in \mathcal{I}_{p, q}:=\left(L_{\sigma}^{p}(G), D\left(A_{S}\right)\right)_{1-1 / p, q}$ there is a unique solution $(u, \pi) \in\left(W^{1, q}\left(\mathbb{R}_{+}, L_{\sigma}^{p}(G)\right) \cap L^{q}\left(\mathbb{R}_{+}, D\left(A_{S}\right)\right)\right) \times L^{q}\left(\mathbb{R}_{+}, \widehat{W}^{1, p}(G)\right)$ of (1.1). This solution satisfies $\pi=0$ and

$$
\left\|\partial_{t} u\right\|_{L^{q}\left(\mathbb{R}_{+}, L^{p}\right)}+\sum_{|\alpha| \leq 2}\left\|\rho^{|\alpha|-2} \partial^{\alpha} u\right\|_{\left.L^{q}\left(\mathbb{R}_{+}, L^{p}\right)\right)} \leq C\left(\|f\|_{L^{q}\left(\mathbb{R}_{+}, L^{p}\right)}+\left\|u_{0}\right\|_{\mathcal{I}_{p, q}}\right)
$$

with $C>0$ independent of $f$ and $u_{0}$.

For the proof of Theorem 1.2 we basically follow the strategy in [21, that is, we first consider the Laplace equation subject to perfect slip conditions. In a standard procedure, by employing polar coordinates and Euler transformation, we reduce the Laplace equation on a wedge to a problem on a layer. On the layer we apply the operator sum method as it is performed originally in [26].

The difference to [21] lies in the fact that here we consider the elliptic problem

$$
\left.\begin{array}{rll}
-\Delta u=f & \text { in } \quad G \\
u \cdot \nu=0 & \text { on } \quad \partial G
\end{array}\right\}
$$

instead of the corresponding resolvent problem. The advantage is that for the transformed problem we then have precise knowlege on the spectrum. This, in turn, allows to completely characterize the set of $p$ for which optimal regularity for (1.4) is available. We formulate this in our second main result which also represents the basis for Theorem 1.2 and which we even prove in Kondrat'ev spaces.

Theorem 1.4. Let $1<p<\infty, \theta_{0} \in(0, \pi), \gamma \in \mathbb{R}$, and $\rho=\left|\left(x_{1}, x_{2}\right)\right|$. Then equation (1.4) is for each $f \in L_{\gamma}^{p}\left(G, \mathbb{R}^{2}\right)$ uniquely solvable with a solution u satisfying

$$
\rho^{|\alpha|-2} \partial^{\alpha} u \in L_{\gamma}^{p}\left(G, \mathbb{R}^{2}\right) \quad(|\alpha| \leq 2)
$$

if and only if

$$
2-\frac{2+\gamma}{p} \notin\left\{\frac{k \pi}{\theta_{0}} \pm 1: k \in \mathbb{N}\right\} \cup\{1\} .
$$

Remark 1.5. (a) For $\gamma=0$ condition (1.6) reduces to

$$
2-\frac{2}{p} \notin\left\{1, \frac{\pi}{\theta_{0}}-1, \frac{2 \pi}{\theta_{0}}-1\right\},
$$

see Subsection 2.5. From this we see that for each angle $\theta_{0} \in(0, \pi)$ the case $p=2$ is excluded. On the other hand, from the results obtained in [10] one would expect $\partial^{\alpha} u \in L^{2}\left(G, \mathbb{R}^{2}\right)$ for $|\alpha|=2$. Taking into account Hardy's inequality, by which the lower oder terms in (1.5) can be estimated by the second order terms, this looks curious at a first glance. However, $p=2$ is exactly the case when Hardy's inequality is not valid. Thus, for $p=2$ (1.5) still can fail for one of the lower order terms, although $\partial^{\alpha} u \in L^{2}\left(G, \mathbb{R}^{2}\right),|\alpha|=2$, might be true. For the excluded $p \neq 2$ (1.5) must fail for at least one of the second order terms, since otherwise Hardy's inequality would yield (1.5) to be valid for all terms, see also Remark 2.7(b). We think that this is an effect of the unboundedness of a wedge domain, see also the lines subsequent to Theorem 1.6.

(b) Another curious looking case is given by $\gamma=0$ and $\theta_{0}=\pi / 2$. Then, by reflection arguments the problem on the wedge $G$ can be reduced to $-\Delta u=f$ on $\mathbb{R}^{2}$. This fact implies $\partial^{\alpha} u \in L^{p}\left(G, \mathbb{R}^{2}\right)$, $|\alpha|=2$, to be valid for all $p \in(1, \infty)$. Again this does not contradict the assertion of Theorem 1.4 , 
since in this case (1.7) is reduced to $2-2 / p \notin\{1\}$. Thus, only $p=2$ is excluded and we find ourselves in the situation explained in (a).

It seems that Theorem 1.4 is not contained in the previous literature. This might rely on the fact that due to the boundary conditions (1.4) is a system, whereas in previous literature the Laplace equation is preferably considered as a scalar equation.

In contrast to Theorem 1.2, as a first consequence of Theorem 1.4 we obtain that for the instationary diffusion equation subject to perfect slip $W^{2, p}$ regularity is not available if condition (1.6) is not fulfilled, see Theorem 2.19 below. The point why we nevertheless can prove Theorem 1.2 relies on the fact that the part of $L^{p}$ destroying $W^{2, p}$ regularity is more or less complemented to the space of solenoidal fields $L_{\sigma}^{p}(G)$. By this fact we obtain optimal regularity for the stationary Stokes equations, too.

Theorem 1.6. Let $1<p<\infty$ and $\theta_{0} \in(0, \pi)$. Then for each $f \in L_{\sigma}^{p}(G)$ there exists a unique solution $(u, \pi) \in\left(W^{2, p}\left(G, \mathbb{R}^{2}\right) \cap L_{\sigma}^{p}(G)\right) \times \widehat{W}^{1, p}(G)$ of the stationary version of (1.1). This solution satisfies $\pi=0$ and

$$
\rho^{|\alpha|-2} \partial^{\alpha} u \in L^{p}\left(G, \mathbb{R}^{2}\right) \quad(|\alpha| \leq 2) .
$$

Of course, the Stokes equations subject to perfect slip in 2D can also be considered without taking the path via the Laplace equation, by utilizing its equivalence to a biharmonic equation. The authors of this note, however, also wanted to compare the two equations concerning regularity. In this regard, we find it most interesting that in the underlying situation the outcome for the Stokes equations is better than for the Laplace or diffusion equation, which usually is vice versa by the fact that the Laplacian enjoys much nicer properties than the Stokes operator.

We also want to remark that the appearance of the weight function $\rho$ is essentially due to the unboundedness of a wedge domain. Since the second order derivatives $\nabla^{2} u$ are $L^{p}$-functions on the wedge $G$, the same integrability is valid for $u$ and $\nabla u$ on any bounded neighbourhood of the vertex of G. This follows by standard results, see, e.g., 9, Lemma II.6.1 and Remark II.6.1]. Thus, especially the case $\gamma=0$ can also be regarded as one reply to the question for $W^{2, q}$-regularity for Laplace and Stokes equations subject to perfect slip on a convex domain.

We outline the strategy of the proofs and the organization of this note. Section 2 contains the approach to the Laplace operator and equation. After fixing notation and transforming from a wedge to a layer, in Subsection 2.3 we establish optimal regularity for the transformed problem. This is based on operator sum methods, that is, Kalton-Weis type theorems. Since the transform from a wedge to a layer is a diffeomorphism, this gives instantly Theorem 1.4, as stated in Subsection 2.4. To carry over regularity from the elliptic problem (1.4) to the instationary diffusion equation, it is enough to show optimal regularity for the resolvent problem

$$
\left.\begin{array}{rll}
(1-\Delta) u=h & \text { in } \quad G, \\
\operatorname{curl} u=0, u \cdot \nu=0 & \text { on } \quad \partial G .
\end{array}\right\}
$$

The idea is to regard $u$ as the solution of the elliptic problem (1.4) with right-hand side $f=h-u \in$ $L^{p}\left(G, \mathbb{R}^{2}\right)$. According to Theorem 1.4 we know that this problem has a solution, say $v$, with the regularity given in (1.5). It remains to prove $u=v$. By the outcome given in 21] this is valid for $p>1$ close to 1 . This means, if the solution operators to problems (1.4) and (1.8) are consistent on the scale $\left(L^{p}\left(G, \mathbb{R}^{2}\right)\right)_{1<p<\infty}$, the regularity in (1.5) transfers to the solution $u$ of (1.8) for all $1<p<\infty$. By the equivalence in Theorem 1.4, however, consistency for the solution operator of (1.4) cannot hold on the full scale $\left(L^{p}\left(G, \mathbb{R}^{2}\right)\right)_{1<p<\infty}$. But, as shown in Subsection 2.5, it is consistent on a suitable 
scale of "nice" subspaces. This leads in Subsection 2.6 to optimal regularity for the diffusion equation on the subspaces for all $1<p<\infty$ (see Theorem 2.23).

A major difficulty for the transference of optimal Sobolev regularity to the Stokes equations is given by the fact that the space of solenoidal fields $L_{\sigma}^{p}\left(G, \mathbb{R}^{2}\right)$ is not directly included in the "nice" subspace of $L^{p}$. A crucial issue, taking the major part of Section 3 , is therefore to prove that it can be isomorphically embedded into this subspace. This isomorphic embedding is also valid for the domains of the involved operators, finally leading to Theorem 1.2 and Theorem 1.6 .

\section{The LAPlace operator on a WedGe domain SUbJeCt to PERFECT SLIP}

2.1. Notation. First we introduce the notation used throughout this note. Let $X$ be a Banach space. For $1 \leq p \leq \infty$ and a measure space $(S, \Sigma, \mu)$, we denote by $L^{p}(S, \mu, X)$ the usual Bochner-Lebesgue space. If $1 \leq p \leq \infty$ and $(S, \Sigma, \mu)$ is a complete measure space, then $L^{p}(S, \mu, X)$ is a Banach space. If $\Omega \subset \mathbb{R}^{n}$ is a domain and $\mu$ is the (Borel-) Lebesgue measure, we write $L^{p}(\Omega, X)$. We define the Sobolev space of order $k \in \mathbb{N}_{0}$ as $W^{k, p}\left(\Omega, \mathbb{R}^{n}\right)$, where $W^{0, p}:=L^{p}$.

Let $G \subset \mathbb{R}^{2}$ be the wedge domain defined in (1.2) and let $\rho=\rho\left(x_{1}, x_{2}\right)=\left|\left(x_{1}, x_{2}\right)\right|$. We set

$$
K_{p, \gamma}^{m}\left(G, \mathbb{R}^{2}\right):=\left\{u \in L_{l o c}^{1}\left(G, \mathbb{R}^{2}\right): \rho^{|\alpha|-m} \partial^{\alpha} u \in L_{\gamma}^{p}\left(G, \mathbb{R}^{2}\right),|\alpha| \leq m\right\}
$$

where $\alpha \in \mathbb{N}^{m}$ denotes a multiindex, $\gamma \in \mathbb{R}$, and $L_{\gamma}^{p}\left(G, \mathbb{R}^{2}\right)$ is defined as in (1.3). Then $K_{p, \gamma}^{m}\left(G, \mathbb{R}^{2}\right)$ equipped with

$$
\|u\|_{K_{p, \gamma}^{m}}:=\|u\|_{K_{p, \gamma}^{m}\left(G, \mathbb{R}^{2}\right)}:=\left(\sum_{|\alpha| \leq m}\left\|\rho^{|\alpha|-m} \partial^{\alpha} u\right\|_{L_{\gamma}^{p}\left(G, \mathbb{R}^{2}\right)}^{p}\right)^{1 / p}
$$

is a Banach space. We also set $K_{p}^{m}\left(G, \mathbb{R}^{2}\right):=K_{p, 0}^{m}\left(G, \mathbb{R}^{2}\right)$. Let $1<p<\infty$ with $1 / p+1 / p^{\prime}=1$. If $u \in L^{p}\left(\Omega, \mathbb{R}^{2}\right)$ and $v \in L^{p^{\prime}}\left(\Omega, \mathbb{R}^{2}\right)$ we denote the duality pairing by $(u, v):=(u, v)_{\Omega}:=\int_{\Omega} u v d x$. For a family $\left(x_{j}\right)_{j \geq 1}$ of elements in a linear space $X$, we denote by

$$
\left\langle x_{j}\right\rangle_{j \geq 1}=\left\langle x_{1}, x_{2}, \ldots\right\rangle
$$

its linear hull.

For Banach spaces $X, Y$ the space of bounded linear operators from $X$ to $Y$ is denoted by $\mathscr{L}(X, Y)$, where $\mathscr{L}(X):=\mathscr{L}(X, X)$. The subclass of isomorphisms is denoted by $\mathscr{L}_{i s}(X, Y)$ or $\mathscr{L}_{i s}(X)$, respectively. If $X^{\prime}$ is the dual space of $X$, then we use for the corresponding duality pairing the notation

$$
\left\langle x^{\prime}, x\right\rangle_{X^{\prime}, X}, \quad x \in X, x^{\prime} \in X^{\prime} .
$$

We denote for a linear operator $A$ in $X$ domain and range by $D(A)$ and $R(A)$. Its spectrum, point spectrum, and resolvent set are written as $\sigma(A), \sigma_{p}(A)$, and $\rho(A)$. We say that an operator $A$ : $D(A) \subset X \rightarrow X$ is sectorial, if $\overline{D(A)}=\overline{R(A)}=X,(0, \infty) \subset \rho(-A)$, and the family $\left(\lambda(\lambda+A)^{-1}\right)_{\lambda>0}$ is uniformly bounded. If the latter family is $\mathcal{R}$-bounded, then we call $A \mathcal{R}$-sectorial. By $\phi_{A}$ and $\phi_{A}^{\mathcal{R}}$ we denote the corresponding spectral and $\mathcal{R}$-angle, respectively [16, 6, 20].

In this note we also employ elements of the $\mathcal{H}^{\infty}$-calculus (e.g. in Theorem $\left[2.3\right.$ ). By $\mathcal{H}^{\infty}(X)$ we denote the class of all operators $A$ in $X$ admitting a bounded $\mathcal{H}^{\infty}$-calculus on $X$. The corresponding $\mathcal{H}^{\infty}$-angle is denoted by $\phi_{A}^{\infty}$. We refer to [16, 6, 20, 27] for an introduction into $\mathcal{H}^{\infty}$-calculus, $\mathcal{R}$ boundedness, and related notions. 
2.2. Transformation of the elliptic linear problem. In this section we transform the elliptic linear problem (1.4) on a two-dimensional wedge domain onto a layer domain of the form $\Omega:=\mathbb{R} \times I$. If $\theta_{0}$ denotes the angle of the wedge $G$ we set $I:=\left(0, \theta_{0}\right)$. In the first step we introduce polar coordinates whereas in the second step we employ the Euler transformation. Last we rescale the appearing terms such that we can work in the transformed setting in unweighted $L^{p}$-spaces.

We write the inverse of the transform to polar coordinates as

$$
\psi_{P}: \mathbb{R}_{+} \times I \rightarrow G, \quad(r, \theta) \mapsto(r \cos \theta, r \sin \theta)=\left(x_{1}, x_{2}\right)
$$

with the associated orthogonal basis

$$
e_{r}=\left(\begin{array}{c}
\cos \theta \\
\sin \theta
\end{array}\right), \quad e_{\theta}=\left(\begin{array}{c}
-\sin \theta \\
\cos \theta
\end{array}\right) .
$$

We identify the orthogonal transformation matrix $\mathcal{O}$ of the components of a vector field as

$$
\mathcal{O}=\left(\begin{array}{cc}
\cos \theta & -\sin \theta \\
\sin \theta & \cos \theta
\end{array}\right)
$$

Next, we employ Euler transformation $r=e^{x}$ in radial direction, where by an abuse of notation we write $x \in \mathbb{R}$ for the new variable. We set

$$
\psi_{E}: \Omega \rightarrow \mathbb{R}_{+} \times I, \quad(x, \theta) \mapsto\left(e^{x}, \theta\right)=:(r, \theta) .
$$

It is not difficult to see that

$$
\psi:=\psi_{P} \circ \psi_{E}: \Omega \rightarrow G
$$

is a diffeomorphism. We set

$$
\Psi u:=u \circ \psi \quad \text { and } \quad \Psi^{-1} v:=v \circ \psi^{-1} .
$$

For $\alpha \in \mathbb{R}$ we also denote the multiplication operator by

$$
M_{\alpha} v:=e^{\alpha x} v .
$$

Analogous to [21] we define pull back resp. push forward by

$$
v:=\Theta_{p}^{*} u:=M_{-\beta_{p}} \mathcal{O}^{-1} \Psi u \quad \text { resp. } u=\Theta_{*}^{p} v=\Psi^{-1} \mathcal{O} M_{\beta_{p}} v
$$

with $\beta_{p} \in \mathbb{R}$ to be chosen later. Then the transformed Laplacian, computed straight forwardly, is given as

with the polynomial

$$
\Theta_{p}^{*}(\Delta u)=e^{-2 x}\left(\begin{array}{l}
r_{p}\left(\partial_{x}\right) v_{x}+\partial_{\theta}^{2} v_{x}-v_{x}-2 \partial_{\theta} v_{\theta} \\
r_{p}\left(\partial_{x}\right) v_{\theta}+\partial_{\theta}^{2} v_{\theta}-v_{\theta}+2 \partial_{\theta} v_{x}
\end{array}\right)
$$

$$
r_{p}\left(\partial_{x}\right):=\partial_{x}^{2}+2 \beta_{p} \partial_{x}+\beta_{p}^{2}
$$

To absorb the factor $e^{-2 x}$, we put

$$
g=\left(g_{x}, g_{\theta}\right):=\widetilde{\Theta}_{p}^{*} f:=e^{2 x} \Theta_{p}^{*} f
$$

so that

$$
\int_{\mathbb{R}}|g(x, \theta)|^{p} d x=\int_{0}^{\infty}\left|r^{2-\beta_{p}} \mathcal{O}^{-1} f\left(\psi_{p}(r, \theta)\right)\right|^{p} \frac{d r}{r} .
$$

Then by the choice $p\left(2-\beta_{p}\right)=\gamma+2$, that is

$$
\beta_{p}=2-\frac{2+\gamma}{p}
$$


we see that in the transformed setting we can work in an unweighted $L^{p}$-space, see [26, 21]. Notice that by this choice of $\beta_{p}$ also pull back and push forward depend on $p$, i.e., the corresponding families are not consistent in $p$.

Finally, we transform the boundary conditions $\nu \cdot u=0$, rot $u=0$ on $\partial G$ of the problem (1.4) to the result that

$$
\partial_{\theta} v_{x}=0, \quad v_{\theta}=0 \quad \text { on } \partial \Omega=\mathbb{R} \times\left\{0, \theta_{0}\right\} .
$$

Summarizing, we receive the following transformed problem on $\Omega=\mathbb{R} \times I$ :

$$
\left.\begin{array}{rl}
r_{p}\left(\partial_{x}\right) v_{x}+\partial_{\theta}^{2} v_{x}-v_{x}-2 \partial_{\theta} v_{\theta}=g_{x} & \text { in } \Omega, \\
r_{p}\left(\partial_{x}\right) v_{\theta}+\partial_{\theta}^{2} v_{\theta}-v_{\theta}+2 \partial_{\theta} v_{x}=g_{\theta} & \text { in } \Omega, \\
\partial_{\theta} v_{x}=0, \quad v_{\theta}=0 \quad \text { on } \partial \Omega .
\end{array}\right\}
$$

2.3. Optimal elliptic regularity for the transformed problem. Here we consider problem (2.5). In this subsection we frequently identify $L^{p}\left(\Omega, \mathbb{R}^{2}\right)$ with its isometrically isomorphic version $L^{p}\left(\mathbb{R}, L^{p}\left(I, \mathbb{R}^{2}\right)\right)$, often without further notice. We introduce the operators associated to the single parts in (2.5):

(1) Let $r_{p}$ be the polynomial given in (2.2). We define $\mathcal{T}_{p, x}$ in $L^{p}(\mathbb{R})$ by means of

$$
\mathcal{T}_{p, x} u:=r_{p}\left(\partial_{x}\right) u, \quad u \in D\left(\mathcal{T}_{p, x}\right):=W^{2, p}(\mathbb{R}) .
$$

The spectrum of $\mathcal{T}_{p, x}$ is given by the parabola $r_{p}(i \mathbb{R})$ which is symmetric about the real axis, open to the left and has its intersection point with the $x$-axis at $\beta_{p}^{2}$ with $\beta_{p}$ as in (2.4). It is straight forward to show that $-\mathcal{T}_{p, x}+b \in \mathcal{H}^{\infty}\left(L^{p}(\mathbb{R})\right)$ for $b>\beta_{p}^{2}$ with $\phi_{-\mathcal{T}_{p, x}+b}^{\infty}<\pi / 2$, e.g., by the use of Fourier transform, see also [26, 21]. By means of operator-valued Fourier multiplier results [33, 6, 20] these facts obviously transfer to the vector-valued version on $L^{p}\left(\mathbb{R}, L^{p}\left(I, \mathbb{R}^{2}\right)\right)$ given as

$$
T_{p, x} u:=\mathcal{T}_{p, x} u, \quad u \in D\left(T_{x}\right):=W^{2, p}\left(\mathbb{R}, L^{p}\left(I, \mathbb{R}^{2}\right)\right) .
$$

(2) We define $\mathcal{T}_{p, \theta}$ in $L^{p}\left(I, \mathbb{R}^{2}\right)$ by

$$
\mathcal{T}_{p, \theta} v:=\left(\begin{array}{cc}
\partial_{\theta}^{2}-1 & -2 \partial_{\theta} \\
2 \partial_{\theta} & \partial_{\theta}^{2}-1
\end{array}\right) v
$$

on $D\left(\mathcal{T}_{p, \theta}\right):=\left\{v=\left(v_{x}, v_{\theta}\right) \in W^{2, p}\left(I, \mathbb{R}^{2}\right): \partial_{\theta} v_{x}=0, v_{\theta}=0\right.$ on $\left.\partial I\right\}$. It is also straight forward to identify

$$
\sigma\left(\mathcal{T}_{p, \theta}\right)=\sigma_{p}\left(\mathcal{T}_{p, \theta}\right)=\left\{-\left(\frac{k \pi}{\theta_{0}} \pm 1\right)^{2}: k \in \mathbb{N}\right\} \cup\{-1\}
$$

as its spectrum with corresponding eigenfunctions $\left(v_{x}^{k}, v_{\theta}^{k}\right)^{\tau}$, where

$$
v_{x}^{k}(\theta):=\cos \left(\frac{k \pi}{\theta_{0}} \theta\right), \quad v_{\theta}^{k}(\theta):= \pm \sin \left(\frac{k \pi}{\theta_{0}} \theta\right), \quad k \in \mathbb{N}_{0}, \theta \in I,
$$

see also [21]. Note that $\mathcal{T}_{p, \theta}$ is self-adjoint in $L^{2}\left(I, \mathbb{R}^{2}\right)$. Hence the eigenfunctions represent a basis of $L^{2}\left(I, \mathbb{R}^{2}\right)$. We denote by $\left(\lambda_{i}\right)_{i \in \mathbb{N}_{0}}$ the set of eigenvalues, i.e., $\left(\lambda_{i}\right)_{i \in \mathbb{N}_{0}}=\sigma\left(\mathcal{T}_{p, \theta}\right)$ such that $\lambda_{0}=-1$ and $\lambda_{1}>\lambda_{2}>\ldots$. Setting $e_{0}:=\left(1 / \sqrt{\theta_{0}}, 0\right)^{\tau}$ and $e_{i}:=\frac{\widetilde{e_{i}}}{\sqrt{\theta_{0}}}$ for $i \in \mathbb{N}$ where $\widetilde{e}_{i}$ denotes the eigenfunction to the eigenvalue $\lambda_{i}$, we have

$$
\left(e_{i}, e_{j}\right)=\frac{1}{\theta_{0}} \int_{0}^{\theta_{0}} \widetilde{e_{i}} \cdot \widetilde{e_{j}} d \theta=\delta_{i j}
$$


By Fourier series techniques it is also standard to prove that $-\mathcal{T}_{p, \theta}$ admits an $\mathcal{H}^{\infty}$-calculus on $L^{p}\left(I, \mathbb{R}^{2}\right)$ with $\phi_{-\mathcal{T}_{p, \theta}}^{\infty}=0$. The same properties remain valid for the canonical extension to $L^{p}\left(\mathbb{R}, L^{p}\left(I, \mathbb{R}^{2}\right)\right)$ denoted by

$$
T_{p, \theta} v:=\mathcal{T}_{p, \theta} v, \quad D\left(T_{p, \theta}\right):=L^{p}\left(\mathbb{R}, D\left(\mathcal{T}_{p, \theta}\right)\right) .
$$

Optimal regularity for (2.5) is then reduced to invertibility of the operator

$$
T_{p}:=T_{p, x}+T_{p, \theta}: D\left(T_{p, x}\right) \cap D\left(T_{p, \theta}\right) \rightarrow L^{p}\left(\Omega, \mathbb{R}^{2}\right)
$$

if we can also show that

$$
\begin{aligned}
D\left(T_{p}\right) & :=\left\{v=\left(v_{x}, v_{\theta}\right) \in W^{2, p}\left(\mathbb{R} \times I, \mathbb{R}^{2}\right), \partial_{\theta} v_{x}=v_{\theta}=0 \text { on } \partial \Omega\right\} \\
& =D\left(T_{p, x}\right) \cap D\left(T_{p, \theta}\right) .
\end{aligned}
$$

The proof of these facts requires some preparation. Let

$$
P_{m, p}^{c} u:=\Sigma_{i=1}^{m}\left(u, e_{i}\right) e_{i}
$$

be the projection of $u \in L^{p}\left(I, \mathbb{R}^{2}\right)$ to $\left\langle e_{1}, \ldots, e_{m}\right\rangle$. We set $P_{m, p}:=1-P_{m, p}^{c}$ and $E_{m}^{p}:=P_{m, p}\left(L^{p}\left(I, \mathbb{R}^{2}\right)\right)$, i.e., $E_{m}^{p}$ is the complement to $\left\langle e_{1}, \ldots, e_{m}\right\rangle$. Note that $\left(P_{m, p}\right)_{1<p<\infty}$ is a consistent family. By this fact we omit the index $p$ and write just $P_{m}$. We denote the extension of $P_{m}$ to $L^{p}\left(\mathbb{R}, L^{p}\left(I, \mathbb{R}^{2}\right)\right)$ by $\mathbb{P}_{m}$. Obviously then $\mathbb{P}_{m} \in \mathscr{L}\left(L^{p}\left(\Omega, \mathbb{R}^{2}\right)\right)$ is a projector as well and we have

$$
L^{p}\left(\Omega, \mathbb{R}^{2}\right)=L^{p}\left(\mathbb{R},\left\langle e_{1}, \ldots, e_{m}\right\rangle\right) \oplus L^{p}\left(\mathbb{R}, E_{m}^{p}\right) .
$$

The following properties are obvious.

Lemma 2.1. Let $T_{p, x}$ and $T_{p, \theta}$ in $L^{p}\left(\Omega, \mathbb{R}^{2}\right)$ for $1<p<\infty$ be defined as above and let $b>\beta_{p}^{2}$ with $\beta_{p}$ as given in 2.4. Then we have

(1) $\mathbb{P}_{m} u \in D\left(T_{p, i}\right)$ and $\mathbb{P}_{m} T_{p, i} u=T_{p, i} \mathbb{P}_{m} u$ for $u \in D\left(T_{p, i}\right)$ and $i \in\{\theta, x\}$;

(2) $-T_{p, x}+b,-T_{p, \theta} \in \mathcal{H}^{\infty}\left(L^{p}\left(\mathbb{R}, E_{m}^{p}\right)\right) \cap \mathcal{H}^{\infty}\left(L^{p}\left(\mathbb{R},\left\langle e_{1}, \ldots, e_{m}\right\rangle\right)\right)$ with the corresponding angles $\phi_{-T_{p, x}+b}^{\infty}<\frac{\pi}{2}$ and $\phi_{-T_{p, \theta}}^{\infty}=0$;

(3) $\mathbb{P}_{m} R\left(\lambda, T_{p, i}\right)=R\left(\lambda, T_{p, i}\right) \mathbb{P}_{m}$ for $\lambda \in \rho\left(T_{p, i}\right)$ and $i \in\{\theta, x\}$;

(4) $\left(\lambda-T_{p, x}\right)^{-1}\left(\mu-T_{p, \theta}\right)^{-1}=\left(\mu-T_{p, \theta}\right)^{-1}\left(\lambda-T_{p, x}\right)^{-1}$ for $\lambda \in \rho\left(T_{p, x}\right)$ and $\mu \in \rho\left(T_{p, \theta}\right)$.

The domains of the Operators $T_{p, x}$ and $T_{p, \theta}$ in the subspace $L^{p}\left(\mathbb{R}, E_{m}^{p}\right)$ are defined as

$$
\begin{aligned}
& D_{m}\left(T_{p, x}\right):=D\left(T_{p, x}\right) \cap L^{p}\left(\mathbb{R}, E_{m}^{p}\right) \quad \text { and } \\
& D_{m}\left(T_{p, \theta}\right):=D\left(T_{p, \theta}\right) \cap L^{p}\left(\mathbb{R}, E_{m}^{p}\right)
\end{aligned}
$$

respectively. The assertions of Lemma 2.1 then easily yield

Corollary 2.2. The operator $\mathbb{P}_{m}$ is a projector on $D\left(T_{p, i}\right)$ and we have

(1) $D_{m}\left(T_{p, i}\right)=\mathbb{P}_{m}\left(D\left(T_{p, i}\right)\right)$,

(2) $D\left(T_{p, i}\right)=D_{m}\left(T_{p, i}\right) \oplus\left(1-\mathbb{P}_{m}\right) D\left(T_{p, i}\right)$

for $i \in\{\theta, x\}$.

We will characterize the invertibility of the operator in (2.7) by employing the operator sum method. More precisely, we apply [24, Proposition 3.5] which is obtained as a consequence of the Kalton-Weis theorem [16, Corollary 5.4]. 
Theorem 2.3. Let $1<p<\infty$ and $\beta_{p}=2-\frac{2+\gamma}{p}$. Then

$$
T_{p, \theta}+T_{p, x} \in \mathscr{L}_{i s}\left(D\left(T_{p, \theta}\right) \cap D\left(T_{p, x}\right), L^{p}\left(\Omega, \mathbb{R}^{2}\right)\right)
$$

if and only if $-\beta_{p}^{2} \notin \sigma\left(T_{p, \theta}\right)$.

Proof. Assume that $-\beta_{p}^{2} \notin \sigma\left(T_{p, \theta}\right)$ and that $b>\beta_{p}^{2}$. The fact that $-\beta_{p}^{2} \notin \sigma\left(T_{p, \theta}\right)$ guarantees

$$
\sigma\left(-T_{p, x}\right) \cap \sigma\left(T_{p, \theta}\right)=\emptyset \text {. }
$$

We first show that $-T_{p, \theta}-T_{p, x}-\varepsilon \in \mathcal{H}^{\infty}\left(L^{p}\left(\mathbb{R}, E_{m}^{p}\right)\right)$ for some $\varepsilon>0$, which essentially gives the assertion.

To this end, pick $m \in \mathbb{N}_{0}$ so that $-\lambda_{m+1}>b$ with $\lambda_{m+1} \in \sigma\left(T_{p, \theta}\right)$. This implies $\sigma\left(-T_{p, \theta}\right) \subset(b, \infty)$ on $L^{p}\left(\mathbb{R}, E_{m}^{p}\right)$ and hence $0 \in \rho\left(-T_{p, \theta}-b-\varepsilon\right)$ for some $\varepsilon>0$. This fact, Lemma 2.1(2) and a standard perturbation argument for $\mathcal{H}^{\infty}$-calculus [12, Corollary 5.5.5] yield that the shifted operator $-T_{p, \theta}-b-\varepsilon$ still satisfies

$$
-T_{p, \theta}-b-\varepsilon \in \mathcal{H}^{\infty}\left(L^{p}\left(\mathbb{R}, E_{m}^{p}\right)\right) \text { with } \phi_{-T_{p, \theta}-b-\varepsilon}^{\infty}=0 .
$$

Thanks to Lemma 2.1(2), which yields $\phi_{-T_{p, \theta}-b-\varepsilon}^{\infty}+\phi_{-T_{p, x}+b}^{\infty}<\pi$, and to Lemma 2.1(4) we may apply [16. Corollary 5.4] (see also [24, Proposition 3.5]) to the result that

$$
-T_{p, \theta}-T_{p, x}-\varepsilon=-T_{p, \theta}-b-\varepsilon+\left(-T_{p, x}+b\right) \in \mathcal{H}^{\infty}\left(L^{p}\left(\mathbb{R}, E_{m}^{p}\right)\right)
$$

with $\phi_{-T_{p, \theta}-T_{p, x}-\varepsilon}^{\infty} \leq \max \left\{\phi_{-T_{p, \theta}-b}^{\infty}, \phi_{-T_{p, x}+b}^{\infty}\right\}$. Particularly, we obtain $0 \in \rho\left(-T_{p, \theta}-T_{p, x}\right)$, hence

$$
T_{p, \theta}+T_{p, x} \in \mathscr{L}_{i s}\left(D_{m}\left(T_{p, x}\right) \cap D_{m}\left(T_{p, \theta}\right), L^{p}\left(\mathbb{R}, E_{m}^{p}\right)\right) .
$$

For the invertibility of the operator $T_{p, \theta}+T_{p, x}$ on $L^{p}\left(\mathbb{R},\left\langle e_{1}, \ldots, e_{m}\right\rangle\right)$ observe that due to (2.12) we have $\lambda_{i} \in \rho\left(-T_{p, x}\right)$ on $L^{p}\left(\Omega, \mathbb{R}^{2}\right)$ for each $\lambda_{i} \in \sigma_{p}\left(T_{p, \theta}\right)$. Thus

$$
\lambda_{i}+T_{p, x}: L^{p}\left(\mathbb{R},\left\langle e_{1}, \ldots, e_{m}\right\rangle\right) \cap D\left(T_{p, x}\right) \rightarrow L^{p}\left(\mathbb{R},\left\langle e_{1}, \ldots, e_{m}\right\rangle\right)
$$

is invertible. By the fact that

$$
\left(T_{p, x}+T_{p, \theta}\right)^{-1} f=\sum_{i=1}^{m}\left(\lambda_{i}+T_{p, x}\right)^{-1}\left(f, e_{i}\right) e_{i}, f \in L^{p}\left(\mathbb{R},\left\langle e_{1}, \ldots, e_{m}\right\rangle\right),
$$

we conclude that

$$
T_{p, \theta}+T_{p, x} \in \mathscr{L}_{i s}\left(L^{p}\left(\mathbb{R},\left\langle e_{1}, \ldots, e_{m}\right\rangle\right) \cap D\left(T_{p, x}\right), L^{p}\left(\mathbb{R},\left\langle e_{1}, \ldots, e_{m}\right\rangle\right)\right) .
$$

Gathering (2.10), (2.13), and (2.14) we end up with

$$
T_{p, \theta}+T_{p, x} \in \mathscr{L}_{i s}\left(D\left(T_{p, x}\right) \cap D\left(T_{p, \theta}\right), L^{p}\left(\Omega, \mathbb{R}^{2}\right)\right) .
$$

Now, assume that $-\beta_{p}^{2} \in \sigma\left(T_{p, \theta}\right)$. Then the symbol $\lambda+r_{p}(i \xi)$ of the operator $T_{p, \theta}+T_{p, x}$ vanishes exactly at $(\lambda, \xi)=\left(-\beta_{p}^{2}, 0\right)$, where $\lambda \in \sigma\left(T_{p, \theta}\right)$. Thus, $\left(\lambda+r_{p}(i \cdot)\right)^{-1}$ is not bounded, hence not an $L^{p}\left(\mathbb{R}, L^{p}\left(I, \mathbb{R}^{2}\right)\right)$-multiplier. This gives the assertion.

Remark 2.4. An inspection of the proof of Theorem 2.3 shows that we even have that $-T_{p, x}-T_{p, \theta}-\varepsilon \in$ $\mathcal{H}^{\infty}\left(L^{p}\left(\Omega, \mathbb{R}^{2}\right)\right)$ with $\phi_{-T_{p, x}-T_{p, \theta}-\varepsilon}^{\infty}<\pi / 2$ for some $\varepsilon>0$.

To obtain optimal regularity we show (2.8). 
Lemma 2.5. Let $1<p<\infty$. Then we have

$$
D\left(T_{p}\right)=D\left(T_{p, \theta}\right) \cap D\left(T_{p, x}\right) .
$$

Proof. Considering the function $\xi \mapsto \frac{i \xi_{i} \cdot i \xi_{j}}{|\xi|^{2}}|\xi|^{2}$ for $\xi \in \mathbb{R}^{2}$ and applying Mihklin's Multiplier Theorem [29] it is not difficult to see that

$$
W^{2, p}\left(\mathbb{R}^{2}, \mathbb{R}^{2}\right)=L^{p}\left(\mathbb{R}, W^{2, p}\left(\mathbb{R}, \mathbb{R}^{2}\right)\right) \cap W^{2, p}\left(\mathbb{R}, L^{p}\left(\mathbb{R}, \mathbb{R}^{2}\right)\right)
$$

with equivalent norms. The validity of (2.8) is proved via an extension theorem, i.e., via a bounded operator $E: W^{2, p}\left(\Omega, \mathbb{R}^{2}\right) \rightarrow W^{2, p}\left(\mathbb{R}^{2}, \mathbb{R}^{2}\right)$ with $\left.E f\right|_{\Omega}=f$ for all $f \in W^{2, p}\left(\Omega, \mathbb{R}^{2}\right)$. See [1, Theorem 4.26] for the existence of $E$.

2.4. Optimal elliptic regularity for problem (1.4). We next consider equivalence of the problems (1.4) and (2.5). The Laplace operator on the wedge domain is defined as

$$
B_{p} u:=-\Delta u, u \in D\left(B_{p}\right):=\left\{u \in K_{p, \gamma}^{2}\left(G, \mathbb{R}^{2}\right): \operatorname{curl} u=0, \nu \cdot u=0 \text { on } \partial G\right\} .
$$

Observe that the boundary conditions are defined in a local sense. Indeed, each $u \in K_{p, \gamma}^{2}\left(G, \mathbb{R}^{2}\right)$ is locally away from the vertex $(0,0)$ a $W^{2, p}$-function for which the traces are well-defined.

Lemma 2.6. Let $1<p<\infty$. Let $\Theta_{*}^{p}, \widetilde{\Theta}_{*}^{p}, \Theta_{p}^{*}, \widetilde{\Theta}_{p}^{*}$ be defined as in Subsection 2.2. Then we have

$$
\widetilde{\Theta}_{*}^{p} \in \mathscr{L}_{i s}\left(L^{p}\left(\Omega, \mathbb{R}^{2}\right), L_{\gamma}^{p}\left(G, \mathbb{R}^{2}\right)\right), \quad \Theta_{*}^{p} \in \mathscr{L}_{i s}\left(D\left(T_{p}\right), D\left(B_{p}\right)\right)
$$

where $\|\cdot\|_{D\left(B_{p}\right)}=\|\cdot\|_{K_{p, \gamma}^{2}\left(G, \mathbb{R}^{2}\right)}$ and $\|\cdot\|_{D\left(T_{p}\right)}=\|\cdot\|_{W^{2, p}\left(\Omega, \mathbb{R}^{2}\right)} \cdot$

In particular, $u \in D\left(B_{p}\right)$ is the unique solution of (1.4) to the right-hand side $f \in L_{\gamma}^{p}\left(G, \mathbb{R}^{2}\right)$ if and only if $v=\Theta_{p}^{*} u \in D\left(T_{p}\right)$ is the unique solution of (2.5) to the right-hand side $g=\widetilde{\Theta}_{p}^{*} f$.

Proof. By utilizing the transformations given in Subsection 2.2 and by the definition of $\widetilde{\Theta}_{*}^{p}$ and $\Theta_{*}^{p}$, it is straight forward to verify (2.15). Hence problem (1.4) and problem (2.5) are equivalent.

Since $-\beta_{p}^{2} \notin \sigma\left(T_{p, \theta}\right)$ is precisely condition (1.6), Theorem 2.3, Lemma 2.5, and Lemma 2.6 now imply our second main result Theorem 1.4

Remark 2.7. (a) Theorem 1.4 in particular implies that $\left(B_{p}^{-1}\right)_{1<p<\infty}$ cannot be a consistent family on the scale $\left(L^{p}\left(\Omega, \mathbb{R}^{2}\right)\right)_{1<p<\infty}$. Otherwise it would be possible to recover the excluded $p$ subject to condition (1.6) by an interpolation argument. By the equivalence in Theorem 1.4 this, however, is not possible.

(b) Note that for $\gamma=0$ we have

$$
\left.\left.\int_{G}\left|u\left(x_{1}, x_{2}\right) /\right|\left(x_{1}, x_{2}\right)\right|^{2}\right|^{p} d x_{1} d x_{2}=\int_{0}^{\theta_{0}} \int_{\mathbb{R}}\left|e^{-(2-2 / p) x} u(\psi(x, \theta))\right|^{p} d x d \theta .
$$

Thus, employing twice Hardy's inequality on the $x$ integral, the terms $\rho^{|\alpha|-2} \partial^{\alpha} u$ for $|\alpha| \leq 1$ can be estimated by the second order terms. This, however, does only work provided $2-|\alpha|-2 / p \neq 0$ which means at the end that $p \neq 2$, since otherwise Hardy's inequality is not applicable. As a consequence, Theorem 1.4 implies that

$$
\left(\partial_{j} \partial_{k} u\right)_{1 \leq j, k \leq 2} \not \subset L^{p}\left(G, \mathbb{R}^{8}\right),
$$

if condition (1.6) is not satisfied and $p \neq 2$. In the case $p=2$ second order derivatives might belong to $L^{2}\left(G, \mathbb{R}^{2}\right)$, but then at least one of the terms $\rho^{|\alpha|-2} \partial^{\alpha} u,|\alpha| \leq 1$, cannot be in $L^{2}\left(G, \mathbb{R}^{2}\right)$. 
2.5. Consistency of $\left(B_{p}^{-1}\right)_{1<p<\infty}$ on a subscale. Observe that condition (1.6) is always fulfilled if every eigenvalue $\lambda_{i}$ of $T_{p, \theta}$ satisfies

$$
\lambda_{i}<-\left(2-\frac{2+\gamma}{p}\right)^{2}
$$

As our main interest concerns the Stokes equations in $L_{\sigma}^{p}(G)$, from now on we restrict ourselves to the case $\gamma=0$, i.e., to the case of Kondrat'ev weight $\rho^{\gamma} \equiv 1$. Then we have

$$
-\beta_{p}^{2}=-\left(2-\frac{2}{p}\right)^{2} \geq-4 \quad(1<p<\infty) .
$$

From (2.6) it is easily seen that

$$
\lambda_{i}<-4 \quad(i \geq 3) .
$$

Thus, relation (2.16) remains true for all $\lambda_{i} \in \sigma\left(T_{p, \theta}\right)$ with $i \geq 3$.

As we will see later (Proposition 3.2), excluding the eigenfunctions $e_{0}, e_{1}, e_{2}$ to the eigenvalues $\lambda_{0}$, $\lambda_{1}, \lambda_{2}$ of the transformed operator $T_{p, \theta}$, will play no significant role for the Stokes equations. Roughly speaking, this is due to the fact that their linear hull in $L^{p}\left(\Omega, \mathbb{R}^{2}\right)$ does not contain divergence free vector fields. Hence, from now on we consider

$$
L^{p}\left(\mathbb{R}, E_{3}^{p}\right)=\mathbb{P}_{3}\left(L^{p}\left(\Omega, \mathbb{R}^{2}\right)\right)
$$

as the base space for $T_{p}: D_{3}\left(T_{p}\right) \rightarrow L^{p}\left(\mathbb{R}, E_{3}^{p}\right)$ with the projector $\mathbb{P}_{3}$ defined in (2.10) and domain

$$
D_{3}\left(T_{p}\right):=D\left(T_{p}\right) \cap L^{p}\left(\mathbb{R}, E_{3}^{p}\right)=D_{3}\left(T_{p, \theta}\right) \cap D_{3}\left(T_{p, x}\right),
$$

with $D_{3}\left(T_{p, \theta}\right)$ and $D_{3}\left(T_{p, x}\right)$ as given in (2.11). As an immediate consequence of Theorem 2.3 (and its proof for $m=3$, in particular (2.13) ) we obtain

Corollary 2.8. We have $T_{p} \in \mathscr{L}_{i s}\left(D_{3}\left(T_{p}\right), L^{p}\left(\mathbb{R}, E_{3}^{p}\right)\right)$ for all $1<p<\infty$.

By Lemma 2.6 $\widetilde{\Theta}_{*}^{p}$ and $\Theta_{*}^{p}$ are isomorphisms with inverse $\widetilde{\Theta}_{p}^{*}$ and $\Theta_{p}^{*}$, respectively. This implies that

$$
\begin{aligned}
& \widetilde{\mathbb{Q}}_{p}:=\widetilde{\Theta}_{*}^{p} \mathbb{P}_{3} \widetilde{\Theta}_{p}^{*} \text { and } \\
& \mathbb{Q}_{p}:=\Theta_{*}^{p} \mathbb{P}_{3} \Theta_{p}^{*}
\end{aligned}
$$

are projectors on $L^{p}\left(G, \mathbb{R}^{2}\right)$ and $D\left(B_{p}\right)$, respectively. We set

$$
\mathbb{L}^{p}:=\widetilde{\mathbb{Q}}_{p}\left(L^{p}\left(G, \mathbb{R}^{2}\right)\right)=\widetilde{\Theta}_{*}^{p} L^{p}\left(\mathbb{R}, E_{3}^{p}\right)
$$

and define the restricted operator

$$
\mathbb{B}_{p}:=\left.B_{p}\right|_{D\left(\mathbb{B}_{p}\right)} \quad \text { with } \quad D\left(\mathbb{B}_{p}\right):=\mathbb{Q}_{p}\left(D\left(B_{p}\right)\right)=\Theta_{*}^{p} D_{3}\left(T_{p}\right) .
$$

Notice that, unless its meaning is given otherwise, in what follows we understand the multiplication operator $M_{\alpha} v:=e^{\alpha x} v$ for $\alpha \in \mathbb{R}$ as an operator $M_{\alpha}: F \rightarrow M_{\alpha}(F)$ for a function space $F$. It is clear that $M_{\alpha}$ is injective for all appearing function spaces $F$. Equipping $M_{\alpha}(F)$ with its canonical norm, we even have $M_{\alpha} \in \mathscr{L}_{i s}\left(F, M_{\alpha}(F)\right)$ and $M_{\alpha}^{-1}=M_{-\alpha}$. Furthermore, if $T \in \mathscr{L}(F)$ commutes with $M_{\alpha}$, then we also have $T \in \mathscr{L}\left(M_{\alpha}(F)\right)$.

By construction it follows

Proposition 2.9. Let $1<p<\infty$. Then we have

(1) The scale $\left(\widetilde{\mathbb{Q}}_{p}\right)_{1<p<\infty}$ is consistent on $\left(L^{p}\left(G, \mathbb{R}^{2}\right)\right)_{1<p<\infty}$ and the scale $\left(\mathbb{Q}_{p}\right)_{1<p<\infty}$ on $\left(D\left(B_{p}\right)\right)_{1<p<\infty}$; 
(2) $\widetilde{\mathbb{Q}}_{p} v=\mathbb{Q}_{p} v$ for $v \in D\left(B_{p}\right) \cap L^{p}\left(G, \mathbb{R}^{2}\right)$;

(3) $B_{p} \mathbb{Q}_{p}=\widetilde{\mathbb{Q}}_{p} B_{p}$

(4) $\mathbb{B}_{p} \in \mathscr{L}_{\text {is }}\left(D\left(\mathbb{B}_{p}\right), \mathbb{L}^{p}\right)$.

In particular, for every $f \in \mathbb{L}^{p}$ there is a unique solution $u \in D\left(\mathbb{B}_{p}\right)$ of (1.4).

Proof. (1) Obviously we have

$$
M_{\alpha} \mathbb{P}_{3} v=\mathbb{P}_{3} M_{\alpha} v \quad\left(v \in C_{c}^{\infty}\left(\mathbb{R}, D\left(\mathcal{T}_{p, \theta}\right)\right), \alpha \in \mathbb{R}\right)
$$

with $\mathcal{T}_{p, \theta}$ as defined in the beginning of Subsection 2.3. From Lemma 2.5 and Lemma A.1 we infer that $C_{c}^{\infty}\left(\mathbb{R}, D\left(\mathcal{T}_{p, \theta}\right)\right)$ is dense in $D\left(T_{p}\right)$. Thus equality (2.18) extends to $v \in D\left(T_{p}\right)$. By the definition of $\Theta_{*}^{p}$ and $\Theta_{p}^{*}($ see (2.1) $)$ this implies

$$
\mathbb{Q}_{p} u=\Psi^{-1} \mathcal{O} M_{\beta_{p}} \mathbb{P}_{3} M_{-\beta_{p}} \mathcal{O}^{-1} \Psi u=\Psi^{-1} \mathcal{O P}_{3} \mathcal{O}^{-1} \Psi u \quad\left(u \in D\left(B_{p}\right)\right) .
$$

By the fact that all operators on the right-hand side do not depend on $p$ we obtain consistency of $\left(\mathbb{Q}_{p}\right)_{1<p<\infty}$. The consistency of $\left(\widetilde{\mathbb{Q}}_{p}\right)_{1<p<\infty}$ is completely analogous.

(2) For $u \in D\left(B_{p}\right) \cap L^{p}\left(\Omega, \mathbb{R}^{2}\right)$ we deduce similarly as in (2.19) that

$$
\begin{aligned}
\mathbb{Q}_{p} u & =\Psi^{-1} \mathcal{O} M_{\beta_{p}} \mathbb{P}_{3} M_{-\beta_{p}} \mathcal{O}^{-1} \Psi u=\Psi^{-1} \mathcal{O} \mathbb{P}_{3} \mathcal{O}^{-1} \Psi u \\
& =\Psi^{-1} \mathcal{O} M_{\beta_{p}+2} \mathbb{P}_{3} M_{-\beta_{p}-2} \mathcal{O}^{-1} \Psi u=\widetilde{\mathbb{Q}}_{p} u .
\end{aligned}
$$

(3) Thanks to Lemma 2.1 we have

$$
B_{p} \mathbb{Q}_{p}=\widetilde{\Theta}_{*}^{p} T_{p} \Theta_{p}^{*} \Theta_{*}^{p} \mathbb{P}_{3} \Theta_{p}^{*}=\widetilde{\Theta}_{*}^{p} \mathbb{P}_{3} T_{p} \Theta_{p}^{*}=\widetilde{\mathbb{Q}}_{p} B_{p}
$$

(4) This is a consequence of representation

$$
\mathbb{B}_{p}=\widetilde{\Theta}_{*}^{p} T_{p} \Theta_{p}^{*} \quad \text { on } \quad D\left(\mathbb{B}_{p}\right),
$$

Lemma 2.6, Corollary 2.8, and the definition of $\mathbb{L}^{p}, D\left(\mathbb{B}_{p}\right)$.

As for the projector $\mathbb{P}_{3}$ before, due to the consistency we write from now on $\mathbb{Q}$ and $\widetilde{\mathbb{Q}}$, i.e., we omit the subscript $p$.

Next, we show consistency of the family $\left(\mathbb{B}_{p}^{-1}\right)_{1<p<\infty}$ on the subscale $\left(\mathbb{L}^{p}\right)_{1<p<\infty}$. Observe that the operator $\mathbb{B}_{p}^{-1}$ is represented as

$$
\mathbb{B}_{p}^{-1}=\left.\Theta_{*}^{p} T_{p}^{-1} \widetilde{\Theta}_{p}^{*}\right|_{\mathbb{L}^{p}}
$$

So, for consistency we need to prove that the right-hand side above does not depend on $p$. Note, however, that the single components $\Theta_{*}^{p}, T_{p}^{-1}, \widetilde{\Theta}_{p}^{*}$ do depend on $p$. Merely their combination can be consistent. For this purpose we first show

Lemma 2.10. Let $1<p \leq q<\infty$ and $\beta_{p}=2-2 / p$. For $f \in C_{c}^{\infty}\left(\mathbb{R}, E_{3}^{q}\right)$ we have

$$
T_{p}^{-1} e^{\left(\beta_{q}-\beta_{p}\right) x} f=e^{\left(\beta_{q}-\beta_{p}\right) x} T_{q}^{-1} f .
$$

Proof. First note that $f \in C_{c}^{\infty}\left(\mathbb{R}, E_{3}^{q}\right)$ and $p \leq q$ yield

$$
e^{\left(\beta_{q}-\beta_{p}\right) x} f \in C_{c}^{\infty}\left(\mathbb{R}, E_{3}^{q}\right) \subset L^{p}\left(\mathbb{R}, E_{3}^{p}\right) .
$$

Hence the application of $T_{p}^{-1}$ to this quantity is defined. Also recall that

$$
T_{p} v=T_{p, \theta} v+T_{p, x} v=\mathcal{T}_{p, \theta} v+\mathcal{T}_{p, x} v=\mathcal{T}_{p, \theta} v+\left(\partial_{x}+\beta_{p}\right)^{2} v
$$


We observe that

$$
\left(\partial_{x}+\beta_{q}\right)^{2} e^{-\left(\beta_{q}-\beta_{p}\right) x}=e^{-\left(\beta_{q}-\beta_{p}\right) x}\left(\partial_{x}+\beta_{p}\right)^{2}
$$

implies that

$$
e^{\left(\beta_{q}-\beta_{p}\right) x} T_{q} e^{-\left(\beta_{q}-\beta_{p}\right) x} v=T_{p} v \quad\left(v \in C_{c}^{\infty}\left(\mathbb{R}, D_{3}\left(\mathcal{T}_{\theta, q}\right)\right)\right),
$$

as an equality in $C_{c}^{\infty}\left(\mathbb{R}, E_{3}^{p}\right)$. Here we set $D_{3}\left(\mathcal{T}_{\theta, q}\right)=D\left(\mathcal{T}_{\theta, q}\right) \cap E_{3}^{q}$ and notice that the assertions of Corollary 2.2 also hold for $\mathcal{T}_{\theta, q}$.

For $v \in C_{c}^{\infty}\left(\mathbb{R}, D_{3}\left(\mathcal{T}_{\theta, p}\right)\right) \hookrightarrow C_{c}^{\infty}\left(\mathbb{R}, E_{3}^{q}\right)$ (Sobolev embedding) we set

$$
v_{k}:=k\left(k+T_{q, \theta}\right)^{-1} v \in C_{c}^{\infty}\left(\mathbb{R}, D_{3}\left(\mathcal{T}_{\theta, q}\right)\right), \quad k \in \mathbb{N} .
$$

By the sectoriality of $T_{q, \theta}$ we obtain $v_{k} \rightarrow v$ in $D_{3}\left(T_{p}\right)$. Hence equality (2.22) extends to $v \in$ $C_{c}^{\infty}\left(\mathbb{R}, D_{3}\left(\mathcal{T}_{\theta, p}\right)\right)$. Setting $X=D_{3}\left(\mathcal{T}_{\theta, p}\right), Y=E_{3}^{p}, k=0$, and $\ell=2$ in Lemma A.1, we see that (2.22) extends to all $v \in D_{3}\left(T_{p}\right)$.

As before, for $\alpha \in \mathbb{R}$ we set $M_{\alpha} v=e^{\alpha x} v$. For $\alpha=\beta_{q}-\beta_{p}$ relation (2.22) then yields

$$
T_{q}=M_{-\alpha} T_{p} M_{\alpha} \in \mathscr{L}_{i s}\left(M_{-\alpha}\left(D_{3}\left(T_{p}\right)\right), M_{-\alpha}\left(L^{p}\left(\mathbb{R}, E_{3}^{p}\right)\right)\right)
$$

with inverse

Thanks to (2.21) we see that

$$
\widetilde{T}_{q}^{-1}=M_{-\alpha} T_{p}^{-1} M_{\alpha}
$$

$$
f=M_{-\alpha}\left(M_{\alpha} f\right) \in M_{-\alpha}\left(L^{p}\left(\mathbb{R}, E_{3}^{p}\right)\right)
$$

for $f \in C_{c}^{\infty}\left(\mathbb{R}, E_{3}^{q}\right)$. Due to this fact it remains to show that $\widetilde{T}_{q}^{-1}$ is consistent with $T_{q}^{-1}$ on $C_{c}^{\infty}\left(\mathbb{R}, E_{3}^{q}\right)$.

For $f \in C_{c}^{\infty}\left(\mathbb{R}, D_{3}\left(\mathcal{T}_{\theta, q}\right)\right)$ we have $M_{\alpha} f \in C_{c}^{\infty}\left(\mathbb{R}, E_{3}^{p}\right)$ and hence $T_{p}^{-1} M_{\alpha} f \in D_{3}\left(T_{p}\right)$. Since (2.22) holds for all $v \in D_{3}\left(T_{p}\right)$ this yields

$$
T_{q} \widetilde{T}_{q}^{-1} f=T_{q} M_{-\alpha} T_{p}^{-1} M_{\alpha} f=M_{-\alpha} \underbrace{M_{\alpha} T_{q} M_{-\alpha}}_{=T_{p}} T_{p}^{-1} M_{\alpha} f=f .
$$

Completely analogous we deduce $\widetilde{T}_{q}^{-1} T_{q} f=f$ for $f \in C_{c}^{\infty}\left(\mathbb{R}, D_{3}\left(\mathcal{T}_{\theta, q}\right)\right)$. Hence $\widetilde{T}_{q}^{-1}=T_{q}^{-1}$ on the set $C_{c}^{\infty}\left(\mathbb{R}, D_{3}\left(\mathcal{T}_{\theta, q}\right)\right)$. By a similar approximation argument as in (2.23) we see that this consistency extends to $C_{c}^{\infty}\left(\mathbb{R}, E_{3}^{q}\right)$. This finally yields the assertion.

In the proof of consistency we also employ the following density property.

Lemma 2.11. Let $1<p \leq q<\infty$. Then we have

$$
\widetilde{\Theta}_{*}^{q}\left(C_{c}^{\infty}\left(\mathbb{R}, E_{3}^{q}\right)\right) \stackrel{d}{\hookrightarrow} \mathbb{L}^{q} \cap \mathbb{L}^{p} .
$$

Proof. Note that

$$
\mathbb{L}^{p}=\widetilde{\Theta}_{*}^{p}\left(L^{p}\left(\mathbb{R}, E_{3}^{p}\right)\right)=\widetilde{\Theta}_{*}^{q} M_{-\alpha}\left(L^{p}\left(\mathbb{R}, E_{3}^{p}\right)\right)
$$

with $M_{-\alpha}$ as defined in the proof of Lemma 2.10 and where $M_{-\alpha}\left(L^{p}\left(\mathbb{R}, E_{3}^{p}\right)\right)$ is again equipped with its canonical norm. This shows that $\widetilde{\Theta}_{*}^{q} \in \mathscr{L}_{i s}\left(M_{-\alpha}\left(L^{p}\left(\mathbb{R}, E_{3}^{p}\right)\right), \mathbb{L}^{p}\right)$ with inverse $\widetilde{\Theta}_{q}^{*}$. Since $\widetilde{\Theta}_{*}^{q} \in \mathscr{L}_{i s}\left(L^{q}\left(\mathbb{R}, E_{3}^{q}\right), \mathbb{L}^{q}\right)$ has the same inverse we conclude that

$$
\widetilde{\Theta}_{*}^{q} \in \mathscr{L}_{i s}\left(L^{q}\left(\mathbb{R}, E_{3}^{q}\right) \cap M_{-\alpha}\left(L^{p}\left(\mathbb{R}, E_{3}^{p}\right)\right), \mathbb{L}^{q} \cap \mathbb{L}^{p}\right) .
$$


Thus, it suffices to show that

$$
C_{c}^{\infty}\left(\mathbb{R}, E_{3}^{q}\right) \stackrel{d}{\hookrightarrow} L^{q}\left(\mathbb{R}, E_{3}^{q}\right) \cap M_{-\alpha}\left(L^{p}\left(\mathbb{R}, E_{3}^{p}\right)\right)=: Y .
$$

To this end, pick $v \in Y$ and choose a bounded interval $J \subset \mathbb{R}$ such that

$$
\left\|v-\chi_{J} v\right\|_{Y}=\left\|v-\chi_{J} v\right\|_{L^{q}\left(\mathbb{R}, E_{3}^{q}\right)}+\left\|M_{\alpha}\left(v-\chi_{J} v\right)\right\|_{L^{p}\left(\mathbb{R}, E_{3}^{p}\right)}<\varepsilon / 2
$$

where $\chi_{J}$ denotes the characteristic function to $J$. By the fact that $\chi_{J} v \in L^{q}\left(J, E_{3}^{q}\right)$ we find $\left(v_{k}\right) \subset$ $C_{c}^{\infty}\left(J, E_{3}^{q}\right)$ such that $v_{k} \rightarrow \chi_{J} v$ in $L^{q}\left(\mathbb{R}, E_{3}^{q}\right)$. Note that, thanks to $E_{3}^{q} \hookrightarrow E_{3}^{p}$, we also have

$$
\left\|M_{\alpha}\left(\chi_{J} v-v_{k}\right)\right\|_{L^{p}\left(\mathbb{R}, E_{3}^{p}\right)} \leq C(J, \alpha)\left\|\chi_{J} v-v_{k}\right\|_{L^{p}\left(J, E_{3}^{q}\right)} \rightarrow 0 \quad(k \rightarrow \infty) .
$$

Consequently, choosing $k$ large enough we can achieve

$$
\left\|v-v_{k}\right\|_{Y} \leq\left\|v-\chi_{J} v\right\|_{Y}+\left\|\chi_{J} v-v_{k}\right\|_{Y}<\varepsilon
$$

and the assertion is proved.

Now we are in position to prove the claimed consistency.

Proposition 2.12. The family $\left(\mathbb{B}_{p}^{-1}\right)_{1<p<\infty}$ is consistent on the subscale $\left(\mathbb{L}^{p}\right)_{1<p<\infty}$.

Proof. Let $p, q \in(1, \infty)$ and without loss of generality $p \leq q$. By the definition of $\Theta_{*}^{p}$, $\widetilde{\Theta}_{p}^{*}$ we have

$$
\Theta_{*}^{p}=\Theta_{*}^{q} e^{-\left(\beta_{q}-\beta_{p}\right) x} \text { and } \widetilde{\Theta}_{p}^{*}=e^{\left(\beta_{q}-\beta_{p}\right) x} \widetilde{\Theta}_{q}^{*} .
$$

Now, pick

$$
f \in \widetilde{\Theta}_{*}^{q}\left(C_{c}^{\infty}\left(\mathbb{R}, E_{3}^{q}\right)\right) \subset \mathbb{L}^{p} \cap \mathbb{L}^{q} .
$$

From (2.20) and Lemma 2.10 we infer

$$
\begin{aligned}
\mathbb{B}_{p}^{-1} f & =\Theta_{*}^{p} T_{p}^{-1} \widetilde{\Theta}_{p}^{*} f \\
& =\Theta_{*}^{q} e^{-\left(\beta_{q}-\beta_{p}\right) x} T_{p}^{-1} e^{\left(\beta_{q}-\beta_{p}\right) x} \widetilde{\Theta}_{q}^{*} f \\
& =\Theta_{*}^{q} T_{q}^{-1} \widetilde{\Theta}_{q}^{*} f=\mathbb{B}_{q}^{-1} f .
\end{aligned}
$$

Proposition 2.9(4) and Lemma 2.11 then yield the assertion.

2.6. The diffusion equation. As before let $\theta_{0} \in(0, \pi)$ be the opening angle of the wedge $G$. For $1<p<\infty$ we define the Laplacian $A_{p}$ subject to perfect slip boundary conditions in $L^{p}\left(G, \mathbb{R}^{2}\right)$ by

$$
\begin{aligned}
A_{p} u & :=-\Delta u, \\
u \in D\left(A_{p}\right) & :=\left\{u \in W^{2, p}\left(G, \mathbb{R}^{2}\right): \operatorname{curl} u=0, \nu \cdot u=0 \text { on } \partial G\right\} \cap K_{p}^{2}\left(G, \mathbb{R}^{2}\right) .
\end{aligned}
$$

Now [21, Theorem 1.1 and Corollary 3.15] gives the following result.

Theorem 2.13. There is a $\delta=\delta\left(\theta_{0}\right)$ such that for $1<p<1+\delta$ the operator $A_{p}$ as defined in 2.24) has maximal regularity on $L^{p}\left(G, \mathbb{R}^{2}\right)$.

Remark 2.14. (a) Note that in [21] the case of a three-dimensional wedge is considered. However, by an inspection of the single steps in the proof it is clear that the case of a two-dimensional wedge is completely analogous.

(b) Also observe that $\delta>0$ can be very small. In fact, the methods in [21] yield the constraint $2-2 / p<\min \left\{1,\left(\pi / \theta_{0}-1\right)\right\}$. Hence we have $\delta\left(\theta_{0}\right) \rightarrow 0$ for $\theta_{0} \rightarrow \pi$. 
(c) From the proof of [21, Theorem 1.1 and Corollary 3.15] it also follows that for each $\lambda \in \rho\left(A_{p}\right)$ the family $\left(\left(\lambda-A_{p}\right)^{-1}\right)_{1<p<1+\delta}$ is consistent on $\left(L^{p}\left(G, \mathbb{R}^{2}\right)\right)_{1<p<1+\delta}$.

By a scaling argument we obtain the following estimate in the homogeneous norm.

Lemma 2.15. Let $1<p<\infty$ and $\rho\left(A_{p}\right) \neq \emptyset$. Then we have

$$
\|u\|_{K_{p}^{2}\left(G, \mathbb{R}^{2}\right)} \leq C\left\|A_{p} u\right\|_{L^{p}\left(G, \mathbb{R}^{2}\right)} \quad\left(u \in D\left(A_{p}\right)\right) .
$$

Proof. We have $\mu-A_{p} \in \mathscr{L}_{i s}\left(D\left(A_{p}\right), L^{p}\left(G, \mathbb{R}^{2}\right)\right)$ for a $\mu \in \mathbb{C}$. We introduce the rescaled function $J_{\lambda} u(x):=\lambda^{-2} u(\lambda x), \lambda>0$, and note that the wedge $G$ is invariant under this scaling. This yields

$$
\begin{aligned}
\|u\|_{K_{p}^{2}\left(G, \mathbb{R}^{2}\right)} & =\lambda^{2 / p}\left\|J_{\lambda} u\right\|_{K_{p}^{2}\left(G, \mathbb{R}^{2}\right)} \leq C \lambda^{2 / p}\left\|\left(A_{p}-\mu\right) J_{\lambda} u\right\|_{L^{p}\left(G, \mathbb{R}^{2}\right)} \\
& \leq C \lambda^{2+2 / p}\left\|J_{\lambda}\left(A_{p}-\mu \lambda^{-2}\right) u\right\|_{L^{p}\left(G, \mathbb{R}^{2}\right)} \\
& =C\left\|\left(A_{p}-\mu \lambda^{-2}\right) u\right\|_{L^{p}\left(G, \mathbb{R}^{2}\right)} \quad\left(\lambda>0, u \in D\left(A_{p}\right)\right) .
\end{aligned}
$$

Letting $\lambda \rightarrow \infty$ yields the assertion.

Remark 2.16. The estimate in Lemma 2.15 implies that $A_{p}$ is injective provided that $\rho\left(A_{p}\right) \neq \emptyset$. This implies that $A_{p}$ is sectorial or $\mathcal{R}$-sectorial, whenever $\left(\lambda\left(\lambda+A_{p}\right)^{-1}\right)_{\lambda>0}$ is uniformly bounded or $\mathcal{R}$-bounded, respectively, see [12].

Next, we show that Theorem 2.13 is still valid on $\mathbb{L}^{p}$. To this end, for $1<p<\infty$ we define $\mathbb{A}_{p}$ as the part of $A_{p}$ in $\mathbb{L}^{p}$, that is

$$
\mathbb{A}_{p} u:=\left.A_{p}\right|_{\mathbb{L}^{p}} u, \quad u \in D\left(\mathbb{A}_{p}\right):=\left\{v \in D\left(A_{p}\right) \cap \mathbb{L}^{p}: A_{p} u \in \mathbb{L}^{p}\right\} .
$$

With the projectors $\mathbb{Q}$ and $\widetilde{\mathbb{Q}}$ as defined in (2.17) we obtain

Lemma 2.17. Let $1<p<\infty$. We have

(1) $D\left(A_{p}\right)=D\left(B_{p}\right) \cap L^{p}\left(G, \mathbb{R}^{2}\right)$ with equivalent norms as well as $\mathbb{Q}=\widetilde{\mathbb{Q}}$ and $A_{p}=B_{p}$ on $D\left(A_{p}\right)$. In particular, $\widetilde{\mathbb{Q}}$ on $L^{p}\left(G, \mathbb{R}^{2}\right)$ is the continuous extension of $\mathbb{Q}$ regarded as a projector on $D\left(A_{p}\right)$.

(2) $\widetilde{\mathbb{Q}} A_{p} u=A_{p} \mathbb{Q} u$ for $u \in D\left(A_{p}\right)$.

(3) $\mathbb{Q}\left(\lambda-A_{p}\right)^{-1} f=\left(\lambda-A_{p}\right)^{-1} \widetilde{\mathbb{Q}} f$ for $f \in L^{p}\left(G, \mathbb{R}^{2}\right)$ and $\lambda \in \rho\left(A_{p}\right)$.

(4) $D\left(\mathbb{A}_{p}\right)=D\left(A_{p}\right) \cap \mathbb{L}^{p}=\mathbb{Q} D\left(A_{p}\right)$.

(5) $\left(\lambda-\mathbb{A}_{p}\right)^{-1}=\left.\left(\lambda-A_{p}\right)^{-1}\right|_{\mathbb{L}^{p}}$ for $\lambda \in \rho\left(A_{p}\right)$.

(6) $\left(\left(\lambda-\mathbb{A}_{p}\right)^{-1}\right)_{1<p<1+\delta}$ is consistent on $\left(\mathbb{L}^{p}\right)_{1<p<1+\delta}$ for $\lambda \in \rho\left(\mathbb{A}_{p}\right)$.

Proof. (1) Note that $D\left(A_{p}\right) \hookrightarrow D\left(B_{p}\right)$ is an immediate consequence of the definition of $D\left(A_{p}\right)$. This gives $B_{p}=A_{p}$ and, by virtue of Proposition $2.9(2)$, also $\mathbb{Q}=\widetilde{\mathbb{Q}}$ on $D\left(A_{p}\right)$. Furthermore, the GagliardoNirenberg inequality and Young's inequality yield

$$
\|\nabla u\|_{p} \leq C\left(\left\|\nabla^{2} u\right\|_{p}+\|u\|_{p}\right) \quad\left(u \in L^{p}\left(G, \mathbb{R}^{2}\right) \cap K_{p}^{2}\left(G, \mathbb{R}^{2}\right)\right) .
$$

Note that the wedge $G$ is an $(\varepsilon, \infty)$ domain and on domains of this type the Gagliardo-Nirenberg inequality holds true [21, Section 5] thanks to the extension operator for homogeneous Sobolev spaces constructed in [14, 2]. This implies

$$
\|u\|_{W^{2, p}} \leq C\left(\|u\|_{p}+\left\|\nabla^{2} u\right\|_{p}\right) \leq C\left(\|u\|_{p}+\|u\|_{K_{p}^{2}}\right)
$$


Thus $D\left(A_{p}\right)=D\left(B_{p}\right) \cap L^{p}\left(G, \mathbb{R}^{2}\right)$ with equivalent norms. From this we easily obtain that $\mathbb{Q}$ is also a projector on $D\left(A_{p}\right)$. Since $D\left(A_{p}\right)$ is dense in $L^{p}\left(G, \mathbb{R}^{2}\right), \widetilde{\mathbb{Q}}$ extends $\mathbb{Q}$ continuously on $L^{p}\left(G, \mathbb{R}^{2}\right)$.

(2) follows directly from (1) and Proposition 2.9(3).

(3) Let $\lambda \in \rho\left(A_{p}\right)$. From (1) and (2) we obtain

$$
\left(\lambda-A_{p}\right) \mathbb{Q}\left(\lambda-A_{p}\right)^{-1} f=\widetilde{\mathbb{Q}} f \quad\left(f \in L^{p}\left(G, \mathbb{R}^{2}\right)\right) .
$$

Applying $\left(\lambda-A_{p}\right)^{-1}$ on both sides yields (3).

(4) Let $u \in D\left(A_{p}\right) \cap \mathbb{L}^{p}$. By (1) we obtain $u=\widetilde{\mathbb{Q}} u=\mathbb{Q} u$, hence $u \in \mathbb{Q} D\left(A_{p}\right)$. Conversely, (1) also yields $\mathbb{Q} D\left(A_{p}\right) \subset D\left(A_{p}\right) \cap \mathbb{L}^{p}$. In view of (2) we next conclude

$$
A_{p} u=A_{p} \mathbb{Q} u=\widetilde{\mathbb{Q}} A_{p} u \in \mathbb{L}^{p},
$$

hence $u \in D\left(\mathbb{A}_{p}\right)$. Since the inclusion $D\left(\mathbb{A}_{p}\right) \subset D\left(A_{p}\right) \cap \mathbb{L}^{p}$ is trivial, the assertion is proved.

(5) Let $\lambda \in \rho\left(A_{p}\right)$. For $f \in \mathbb{L}^{p}$ relations (3) and (4) yield

$$
\left(\lambda-A_{p}\right)^{-1} f=\mathbb{Q}\left(\lambda-A_{p}\right)^{-1} f \in D\left(\mathbb{A}_{p}\right) .
$$

Thus,

which proves $(5)$.

$$
\left(\lambda-\mathbb{A}_{p}\right)\left(\lambda-A_{p}\right)^{-1} f=f
$$

(6) follows from (5) and Remark 2.14(c).

By combining the well-known equivalence of maximal regularity and $\mathcal{R}$-sectoriality [33, Theorem 4.2] with Theorem 2.13, Remark 2.16, and Lemma 2.17 (especially assertion (5)) we obtain

Theorem 2.18. Let $1<p<1+\delta$ with $\delta>0$ as in Theorem 2.13. Then $\mathbb{A}_{p}: D\left(\mathbb{A}_{p}\right) \rightarrow \mathbb{L}^{p}$ with domain

$$
D\left(\mathbb{A}_{p}\right)=\left\{u \in W^{2, p}\left(G, \mathbb{R}^{2}\right): \operatorname{curl} u=0, \nu \cdot u=0 \text { on } \partial G\right\} \cap K_{p}^{2}\left(G, \mathbb{R}^{2}\right) \cap \mathbb{L}^{p}
$$

is $\mathcal{R}$-sectorial with $\phi_{\mathbb{A}_{p}}^{\mathcal{R}}<\pi / 2$. Thus, $\mathbb{A}_{p}$ has maximal regularity on $\mathbb{L}^{p}$.

Our ultimate aim in this subsection is to show that Theorem 2.18, in particular the optimal Sobolev regularity, is available on the full range $1<p<\infty$. Note that this is not true for $A_{p}: D\left(A_{p}\right) \subset$ $L^{p}\left(G, \mathbb{R}^{2}\right) \rightarrow L^{p}\left(G, \mathbb{R}^{2}\right)$ with $D\left(A_{p}\right)$ given in (2.24) as the next result shows.

Theorem 2.19. Let $1<p<\infty$ and $\theta_{0} \in(0, \pi)$ such that condition (1.6) (with $\gamma=0$ ) is not satisfied. Then $\rho\left(A_{p}\right)=\emptyset$. In other words, in this situation for every $\lambda \in \mathbb{C}$ there is an $f \in L^{p}\left(G, \mathbb{R}^{2}\right)$ such that there is no solution $u$ of

$$
\left.\begin{array}{rll}
\lambda u-\Delta u=f & \text { in } & G, \\
\text { curlu }=0, u \cdot \nu=0 & \text { on } & \partial G
\end{array}\right\}
$$

satisfying $u \in K_{p}^{2}\left(G, \mathbb{R}^{2}\right)$. More precisely, if $p \neq 2$ then $\partial^{\alpha} u \notin L^{p}\left(G, \mathbb{R}^{2}\right)$ for some $\alpha$ with $|\alpha|=2$, while for $p=2$ we have $\rho^{|\alpha|-2} \partial^{\alpha} u \notin L^{2}\left(G, \mathbb{R}^{2}\right)$ for some $\alpha$ with $|\alpha|<2$.

Proof. Suppose there exists a complex number $\mu \in \rho\left(A_{p}\right)$. We can assume $\mu \neq 0$, since otherwise this would immediately contradict Theorem 1.4 .

By the scaling argument used in the proof of Lemma 2.15] it easily follows that $\left(\left(\lambda-A_{p} / \mu\right)^{-1}\right)_{\lambda>0}$ is uniformly bounded. Thanks to Remark 2.16 then $A_{p} / \mu$ is sectorial, see [12, in particular it has dense range. For $f \in L^{p}\left(G, \mathbb{R}^{2}\right)$ we hence find $\left(u_{k}\right) \subset D\left(A_{p}\right)$ such that $A_{p} u_{k} \rightarrow f$ in $L^{p}\left(G, \mathbb{R}^{2}\right)$. Due 
to Lemma 2.15 $\left(u_{k}\right)$ is a Cauchy sequence in $K_{p}^{2}\left(G, \mathbb{R}^{2}\right)$ and its limit $u=\lim u_{k}$ satisfies equation (1.4). The fact that $u \in K_{p}^{2}\left(G, \mathbb{R}^{2}\right)$ then contradicts Theorem 1.4. Thus $\rho\left(A_{p}\right)$ must be empty. The additional statement follows from Remark 2.7(b).

Next, we show that the resolvent of $\mathbb{A}_{p}$ in $\mathbb{L}^{p}$ is consistent with its dual resolvent. For this purpose we first identify $\left(\mathbb{L}^{p}\right)^{\prime}$. This, in turn, is connected to the identification of $\mathbb{P}_{3}^{\prime}$ and $\mathbb{Q}^{\prime}$. By this fact, just within the following lemma, we write $\mathbb{P}_{3, p}$ and $\mathbb{Q}_{p}$ again.

Lemma 2.20. Let $1<p<\infty, \beta_{p}=2-2 / p$, and $1 / p+1 / p^{\prime}=1$. Let $\widetilde{\Theta}_{*}^{p}: L^{p}\left(\Omega, \mathbb{R}^{2}\right) \rightarrow L^{p}\left(G, \mathbb{R}^{2}\right)$ be defined as in Subsection 2.2 with inverse $\widetilde{\Theta}_{p}^{*}$ and the projectors $\mathbb{P}_{3, p}$ and $\widetilde{\mathbb{Q}}_{p}$ be defined as in (2.9) (and the subsequent lines) and (2.17) respectively. Then we have

(1) $\left(\widetilde{\Theta}_{*}^{p}\right)^{\prime}=\widetilde{\Theta}_{p^{\prime}}^{*}$ and $\left(\widetilde{\Theta}_{p}^{*}\right)^{\prime}=\widetilde{\Theta}_{*}^{p^{\prime}}$; in particular $\widetilde{\Theta}_{*}^{p}$ is an isometric isomorphism;

(2) $\left(\mathbb{P}_{3, p}\right)^{\prime}=\mathbb{P}_{3, p^{\prime}}$;

(3) $\left(\widetilde{\mathbb{Q}}_{p}\right)^{\prime}=\widetilde{\mathbb{Q}}_{p^{\prime}}$;

(4) $\left(\mathbb{L}^{p}\right)^{\prime}=\mathbb{L}^{p^{\prime}}$ with respect to $(u, v)=\int_{G} u v d x$ in the sense of a Riesz isomorphism.

Proof. (1) Recall that by (2.1) and (2.3) we have $\widetilde{\Theta}_{*}^{p} u=\Psi^{-1} \mathcal{O} M_{\beta_{p}-2} u$ with $\Psi, \mathcal{O}, M_{\beta_{p}-2}$ as defined in Subsection 2.2. Thanks to

$$
\beta_{p}=2-\frac{2}{p}=-\beta_{p^{\prime}}+2
$$

we can calculate

$$
\begin{aligned}
\left(\widetilde{\Theta}_{*}^{p} u, v\right)_{G} & =\int_{G} v(y)\left(\mathcal{O} M_{\beta_{p}-2} u\right)\left(\psi^{-1}(y)\right) d y \\
& =\int_{\Omega} v(\psi(x, \theta))\left(\mathcal{O} M_{\beta_{p}-2} u\right)(x, \theta) e^{2 x} d x d \theta \\
& =\int_{\Omega}\left(M_{-\beta_{p^{\prime}}+2} \mathcal{O}^{-1} \Psi v\right)(x, \theta) u(x, \theta) d x d \theta \\
& =\left(u, \widetilde{\Theta}_{p^{\prime}}^{*} v\right)_{\Omega} \quad\left(u \in L^{p}\left(\Omega, \mathbb{R}^{2}\right), v \in L^{p^{\prime}}\left(G, \mathbb{R}^{2}\right)\right) .
\end{aligned}
$$

Relation $\left(\widetilde{\Theta}_{p}^{*}\right)^{\prime}=\widetilde{\Theta}_{*}^{p^{\prime}}$ then follows since $\widetilde{\Theta}_{p}^{*}=\left(\widetilde{\Theta}_{*}^{p}\right)^{-1}$.

Relation (2) follows immediately by the definition of $\mathbb{P}_{3, p}$ and (3) is a consequence of (1) and (2).

(4) By the fact that $\mathbb{L}^{p}=\widetilde{\mathbb{Q}}_{p} L^{p}\left(G, \mathbb{R}^{2}\right)$ this follows from the symmetry of $\widetilde{\mathbb{Q}}_{p}$ proved in (3) and since $\left(L^{p}\left(G, \mathbb{R}^{2}\right)\right)^{\prime}=L^{p^{\prime}}\left(G, \mathbb{R}^{2}\right)$ with respect to $(\cdot, \cdot)$.

Now, let

$$
\mathbb{A}_{p}^{\prime}: D\left(\mathbb{A}_{p}^{\prime}\right) \subset \mathbb{L}^{p^{\prime}} \rightarrow \mathbb{L}^{p^{\prime}}
$$

be the Banach space dual operator to $\mathbb{A}_{p}$ in $\mathbb{L}^{p}$ for $1<p<1+\delta$. By permanence properties and Theorem 2.18 it follows that also $\mathbb{A}_{p}^{\prime}$ is $\mathcal{R}$-sectorial with $\phi_{\mathbb{A}_{p}^{\prime}}^{\mathcal{R}}=\phi_{\mathbb{A}_{p}}^{\mathcal{R}}<\pi / 2$. At this point, however, we do not know how $D\left(\mathbb{A}_{p}^{\prime}\right)$ looks like. On our way to characterize $D\left(\mathbb{A}_{p}^{\prime}\right)$ we next show consistency of $\left(\lambda-\mathbb{A}_{p}\right)^{-1}$ and $\left(\lambda-\mathbb{A}_{p}^{\prime}\right)^{-1}$ on $\mathbb{L}^{p} \cap \mathbb{L}^{p^{\prime}}$.

Proposition 2.21. Let $1<p<1+\delta$ with $\delta>0$ as in Theorem 2.18 and $1 / p+1 / p^{\prime}=1$. Then

$$
\left(\lambda-\mathbb{A}_{p}\right)^{-1} f=\left(\lambda-\mathbb{A}_{p}^{\prime}\right)^{-1} f \quad\left(f \in \mathbb{L}^{p} \cap \mathbb{L}^{p^{\prime}}, \lambda \in \rho\left(\mathbb{A}_{p}\right) \cap \mathbb{R}\right) .
$$


Proof. Let $\lambda \in \rho\left(\mathbb{A}_{p}\right) \cap \mathbb{R}$. We intent to apply Lemma A.2. Setting $T=\lambda-\mathbb{A}_{p}$, we first have to verify that there exists an embedding $J: D\left(\mathbb{A}_{p}\right) \rightarrow\left(\mathbb{L}^{p}\right)^{\prime}$ with dense range. Observe that, since $D\left(\mathbb{A}_{p}\right) \hookrightarrow W^{2, p}\left(G, \mathbb{R}^{2}\right)$ and $G \subset \mathbb{R}^{2}$, the Sobolev embedding yields

$$
D\left(\mathbb{A}_{p}\right) \stackrel{d}{\hookrightarrow} L^{p^{\prime}}\left(G, \mathbb{R}^{2}\right) \cap \mathbb{L}^{p}=\mathbb{L}^{p^{\prime}} .
$$

Thus $J$ can be chosen essentially as the Riesz isomorphism given in Lemma 2.20(4). However, since we identify $\left(\mathbb{L}^{p}\right)^{\prime}$ with $\mathbb{L}^{p^{\prime}}$ anyway and $T^{\sharp}$ with $\left(\lambda-\mathbb{A}_{p}\right)^{\sharp}$ on $\mathbb{L}^{p^{\prime}}$, that is, with its dual induced by the Riesz isomorphism, we omit $J$ (and hence also $\widetilde{J}$ ) in what follows.

By virtue of Lemma A.2 and (A.1) it then remains to prove that

$$
\lambda-\mathbb{A}_{p} \subset\left(\lambda-\mathbb{A}_{p}\right)^{\sharp},
$$

where $\left(\lambda-\mathbb{A}_{p}\right)^{\sharp}: \mathbb{L}^{p^{\prime}} \rightarrow D\left(\mathbb{A}_{p}\right)^{\prime}$ denotes the dual operator of $\lambda-\mathbb{A}_{p}$ regarded as a bounded operator from $D\left(\mathbb{A}_{p}\right)$ to $\mathbb{L}^{p}$, see Appendix $\mathbb{A}$. To this end, pick $u, v \in D\left(\mathbb{A}_{p}\right)$. Observe that by the fact that $D\left(\mathbb{A}_{p}\right) \hookrightarrow \mathbb{L}^{p} \cap \mathbb{L}^{p^{\prime}}$ all duality pairings appearing below are well-defined. Also note that

$$
\Delta u=\nabla \operatorname{div} u-\operatorname{curl}^{\prime} \operatorname{curl} u,
$$

where $\operatorname{curl}^{\prime} \varphi=\left(\partial_{x_{2}},-\partial_{x_{1}}\right)^{T} \varphi$ for a scalar function $\varphi$. Employing the Gauß theorem and the boundary conditions for $u$ and $v$ we calculate

$$
(\nabla \operatorname{div} u, v)=\int_{\partial G} \nu \cdot v \operatorname{div} u d \sigma-(\operatorname{div} u, \operatorname{div} v)=(u, \nabla \operatorname{div} v)
$$

as well as

$$
\begin{aligned}
\left(\operatorname{curl}^{\prime} \operatorname{curl} u, v\right) & =-\int_{\partial G}\left(\left(v^{2},-v^{1}\right)^{T} \cdot \nu\right) \operatorname{curl} u d \sigma+(\operatorname{curl} u, \operatorname{curl} v) \\
& =\left(u, \operatorname{curl}^{\prime} \operatorname{curl} v\right)
\end{aligned}
$$

This yields

$$
\begin{aligned}
\left\langle T^{\sharp} u, v\right\rangle_{D\left(\mathbb{A}_{p}\right)^{\prime}, D\left(\mathbb{A}_{p}\right)} & =(u,(\lambda+\Delta) v)=((\lambda+\Delta) u, v) \\
& =(T u, v)=\langle T u, v\rangle_{D\left(\mathbb{A}_{p}\right)^{\prime}, D\left(\mathbb{A}_{p}\right)}
\end{aligned}
$$

which proves the claim.

Now we can characterize $D\left(\mathbb{A}_{p}^{\prime}\right)$.

Theorem 2.22. Let $1<p<\infty$ and $1 / p+1 / p^{\prime}=1$. Then we have $\mathbb{A}_{p}^{\prime}=\mathbb{A}_{p^{\prime}}$, i.e., in particular $D\left(\mathbb{A}_{p}^{\prime}\right)=D\left(\mathbb{A}_{p^{\prime}}\right)$ with $D\left(\mathbb{A}_{p^{\prime}}\right)$ as characterized by 2.24) and Lemma 2.17(4). Furthermore, for $\lambda \in$ $\rho\left(\mathbb{A}_{p}\right)$ the family $\left(\left(\lambda-\mathbb{A}_{p}\right)^{-1}\right)_{1<p<\infty}$ is consistent on $\left(\mathbb{L}^{p}\right)_{1<p<\infty}$.

Proof. By definition it is obvious that $\mathbb{A}_{p^{\prime}} \subset \mathbb{A}_{p}^{\prime}$. It is clear that the converse inclusion, particularly the assertion on $D\left(\mathbb{A}_{p}^{\prime}\right)$, is proved, if we can show that

$$
\left(1+\mathbb{A}_{p}\right)^{-1} \in \mathscr{L}_{i s}\left(\mathbb{L}^{p}, D\left(\mathbb{A}_{p}\right)\right)
$$

for every $p \in(1, \infty)$. By Theorem 2.18 relation (2.26) holds for every $1<p<1+\delta$. We take $p$ out of that interval and consider (2.26) for its Hölder conjugated exponent $p^{\prime}$.

Let $f \in \mathbb{L}^{p} \cap \mathbb{L}^{p^{\prime}}$. Then there is a $u \in D\left(\mathbb{A}_{p}^{\prime}\right)$ such that

$$
\left(1+\mathbb{A}_{p}^{\prime}\right) u=f .
$$


By the consistency of the resolvents of $\mathbb{A}_{p}$ and $\mathbb{A}_{p}^{\prime}$ proved in Proposition 2.21 we see that $u \in D\left(\mathbb{A}_{p}\right)$ and that

$$
\left(1+\mathbb{A}_{p}\right) u=f \quad \Leftrightarrow \quad \mathbb{A}_{p} u=f-u=: g \in \mathbb{L}^{p} \cap \mathbb{L}^{p^{\prime}} .
$$

On the other hand, Proposition $2.9(4)$ and the consistency of $\left(\mathbb{B}_{p}^{-1}\right)_{1<p<\infty}$ established in Proposition 2.12 imply that there is an $v \in D\left(\mathbb{B}_{p}\right) \cap D\left(\mathbb{B}_{p^{\prime}}\right)$ such that

$$
\mathbb{B}_{p} v=g .
$$

The fact that $D\left(\mathbb{A}_{p}\right) \subset D\left(\mathbb{B}_{p}\right)$ and $\mathbb{A}_{p}=\mathbb{B}_{p}$ on $D\left(\mathbb{A}_{p}\right)$ (Lemma $\left.2.17(1),(4)\right)$ then gives $u=v$. From this and Lemma 2.17(1) we obtain

$$
\begin{aligned}
\left\|\left(1+\mathbb{A}_{p}^{\prime}\right)^{-1} f\right\|_{D\left(\mathbb{A}_{p^{\prime}}\right)} & =\|u\|_{D\left(\mathbb{A}_{p^{\prime}}\right)} \leq C\left(\|u\|_{p^{\prime}}+\|v\|_{K_{p^{\prime}}^{2}}\right) \\
& \leq C\|f\|_{p^{\prime}}\left(f \in \mathbb{L}^{p} \cap \mathbb{L}^{p^{\prime}}\right) .
\end{aligned}
$$

Since $\mathbb{L}^{p} \cap \mathbb{L}^{p^{\prime}}$ lies dense in $\mathbb{L}^{p^{\prime}}$, relation (2.26) follows for $p^{\prime}$.

According to what we just have proved, Lemma 2.17(6), and Proposition 2.21 the family $\left(\left(1+\mathbb{A}_{p}\right)^{-1}\right)_{p \in I}$ is consistent on $\left(\mathbb{L}^{p}\right)_{p \in I}$ for

$$
I=(1, \infty) \backslash\left[1+\delta,(1+\delta)^{\prime}\right] .
$$

For the remaining $p$ we interpolate. In fact, since $\mathbb{L}^{p}=\widetilde{\mathbb{Q}} L^{p}\left(G, \mathbb{R}^{2}\right)$ complex interpolation and 31, Theorem 1.17.1.1] yield

$$
\left[\mathbb{L}^{p}, \mathbb{L}^{p^{\prime}}\right]_{s}=\mathbb{L}^{q}, \quad \frac{1}{q}=s \frac{1}{p^{\prime}}+(1-s) \frac{1}{p} .
$$

Furthermore, by 31] we also have

$$
\begin{aligned}
W^{2, q}\left(G, \mathbb{R}^{2}\right) & =\left[W^{2, p}\left(G, \mathbb{R}^{2}\right), W^{2, p^{\prime}}\left(G, \mathbb{R}^{2}\right)\right]_{s}, \\
K_{q}^{2}\left(G, \mathbb{R}^{2}\right) & =\left[K_{p}^{2}\left(G, \mathbb{R}^{2}\right), K_{p^{\prime}}^{2}\left(G, \mathbb{R}^{2}\right)\right]_{s} .
\end{aligned}
$$

(Note that the second identity above follows, e.g., from

$$
W^{2, q}\left(\Omega, \mathbb{R}^{2}\right)=\left[W^{2, p}\left(\Omega, \mathbb{R}^{2}\right), W^{2, p^{\prime}}\left(\Omega, \mathbb{R}^{2}\right)\right]_{s},
$$

and an application of Stein's interpolation theorem [32], since the dependence of $\Theta_{*}^{q}, \Theta_{q}^{*}$ on $z=1 / q$ is analytic on a suitable strip in the complex plane.) This shows that

$$
\left(1+\mathbb{A}_{p}\right)^{-1} \in \mathscr{L}\left(\mathbb{L}^{p}, W^{2, q}\left(G, \mathbb{R}^{2}\right) \cap K_{q}^{2}\left(G, \mathbb{R}^{2}\right) \cap \mathbb{L}^{p}\right)
$$

for every $p \in(1, \infty)$. For $f \in \mathbb{L}^{p} \cap \mathbb{L}^{q}$ with $q \in I$, we also see that $\left(1+\mathbb{A}_{p}\right)^{-1} f$ satisfies the boundary conditions included in $D\left(\mathbb{A}_{p}\right)$. By a density argument and boundedness of the corresponding trace operators relation (2.26) follows to be valid for all $p \in(1, \infty)$. This completes the proof.

Thanks to Theorem 2.22 we can generalize Theorem 2.18 to all $p \in(1, \infty)$.

Theorem 2.23. Let $1<p<\infty$. Then $\mathbb{A}_{p}$ with domain

$$
D\left(\mathbb{A}_{p}\right)=\left\{u \in W^{2, p}\left(G, \mathbb{R}^{2}\right): \operatorname{curl} u=0, \nu \cdot u=0 \text { on } \partial G\right\} \cap K_{p}^{2}\left(G, \mathbb{R}^{2}\right) \cap \mathbb{L}^{p}
$$

is $\mathcal{R}$-sectorial on $\mathbb{L}^{p}$ with $\phi_{\mathbb{A}_{p}}^{\mathcal{R}}<\pi / 2$, and hence has maximal regularity on $\mathbb{L}^{p}$. 
Proof. Due to $\mathbb{A}_{p}^{\prime}=\mathbb{A}_{p^{\prime}}$ and Theorem 2.18, the operator $\mathbb{A}_{p}$ with $D\left(\mathbb{A}_{p}\right)$ as stated is $\mathcal{R}$-sectorial with $\phi_{\mathbb{A}_{p}}^{\mathcal{R}}<\pi / 2$ for $p \in I$ with $I$ given in (2.27). Note that injectivity, hence also $\overline{R\left(\mathbb{A}_{p}\right)}=\mathbb{L}^{p}$, follows from Remark 2.16. Since the property of $\mathcal{R}$-sectoriality is invariant under interpolation [15, Theorem 3.23], the result follows by interpolation and the equivalence of maximal regularity and $\mathcal{R}$-sectoriality 33 , Theorem 4.2].

In this subsection we have shown by consistency arguments that regularity for the elliptic operator $\mathbb{B}_{p}$ transfers to the parabolic operator $\partial_{t}+\mathbb{A}_{p}$. The next result, which in principle shows that the converse is true as well, we state also for later purposes.

Proposition 2.24. Let $1<p<\infty$. If $\left(\lambda_{k}\right) \subset \rho\left(\mathbb{A}_{p}\right)$ such that $\lim _{k \rightarrow \infty} \lambda_{k}=0$, then

$$
\lim _{k \rightarrow \infty}\left(\lambda_{k}-\mathbb{A}_{p}\right)^{-1}=\mathbb{B}_{p}^{-1} \quad \text { in } \quad \mathscr{L}\left(\mathbb{L}^{p}, K_{p}^{2}\left(G, \mathbb{R}^{2}\right)\right) .
$$

In particular, $D\left(\mathbb{A}_{p}\right)$ is dense in $D\left(\mathbb{B}_{p}\right)$.

Proof. Pick $f \in \mathbb{L}^{p}$. For $\ell \in \mathbb{N}$ by the resolvent identity, Lemma 2.15, and since $\mathbb{A}_{p}$ is sectorial we obtain

$$
\begin{aligned}
& \left\|\left(\lambda_{k+\ell}-\mathbb{A}_{p}\right)^{-1} f-\left(\lambda_{k}-\mathbb{A}_{p}\right)^{-1} f\right\|_{K_{p}^{2}} \\
& \leq C\left\|\left(\lambda_{k+\ell}-\lambda_{k}\right)\left(\lambda_{k}-\mathbb{A}_{p}\right)^{-1} \mathbb{A}_{p}\left(\lambda_{k+\ell}-\mathbb{A}_{p}\right)^{-1} f\right\|_{p} \\
& \leq C\left\|\left(\lambda_{k+\ell} / \lambda_{k}-1\right) f\right\|_{p} \rightarrow 0 \quad(k \rightarrow \infty) .
\end{aligned}
$$

Thus $\left(\lambda_{k}-\mathbb{A}_{p}\right)^{-1} f \rightarrow v$ in $D\left(\mathbb{B}_{p}\right)$. The fact that $\mathbb{B}_{p} \in \mathscr{L}\left(D\left(\mathbb{B}_{p}\right), \mathbb{L}^{p}\right)$, Lemma 2.17(1), and again sectoriality of $\mathbb{A}_{p}$ yield

$$
\mathbb{B}_{p} v=\lim _{k \rightarrow \infty} \mathbb{A}_{p}\left(\lambda_{k}-\mathbb{A}_{p}\right)^{-1} f=f,
$$

hence $v=\mathbb{B}_{p}^{-1} f$.

\section{The Stokes equations}

In this section, we consider the Stokes problem (1.1). We introduce the space of solenoidal vector fields. For $1<p<\infty$ and $1 / p+1 / p^{\prime}=1$ we set

$$
L_{\sigma}^{p}(G):=\left\{u \in L^{p}\left(G, \mathbb{R}^{2}\right): \int_{G} u \cdot \nabla \varphi d\left(x_{1}, x_{2}\right)=0 \quad\left(\varphi \in \widehat{W}^{1, p^{\prime}}(G)\right)\right\},
$$

where

$$
\widehat{W}^{1, p^{\prime}}(G):=\left\{\varphi \in L_{l o c}^{1}(G): \nabla \varphi \in L^{p^{\prime}}\left(G, \mathbb{R}^{2}\right)\right\} .
$$

Since $C_{c}^{\infty}(G) \subset \widehat{W}^{1, p}(G)$, it is evident that $u \in L_{\sigma}^{p}(G)$ satifies the condition $\operatorname{div} u=0$ in the sence of distributions. Moreover $\nu \cdot u$ is well-defined in the trace space (Slobodeckii space) $W_{p}^{-1 / p}(\mathcal{O})$ for all bounded domains $\mathcal{O}$ with $\overline{\mathcal{O}} \subset \partial G \backslash\{(0,0)\}$. This yields that the boundary condition $u \cdot \nu=0$ is fulfilled in a local sense away from 0 .

We define the Stokes operator $A_{S}$ as the part of $A_{p}$ in $L_{\sigma}^{p}(G)$, i.e.,

$$
\begin{aligned}
A_{S} u & :=\left.A_{p}\right|_{L_{\sigma}^{p}(G)} u, \quad u \in D\left(A_{S}\right), \\
D\left(A_{S}\right) & :=\left\{v \in D\left(A_{p}\right) \cap L_{\sigma}^{p}(G): A_{p} v \in L_{\sigma}^{p}(G)\right\} .
\end{aligned}
$$


Note that for the boundary conditions considered here, as a well-known fact, the Helmholtz projection and the Laplacian commute, and the projection does not appear in the definition of $A_{S}$. The next lemma justifies this definition of the Stokes operator.

Lemma 3.1. Let $1<p<\infty$. Then

$$
D\left(A_{S}\right)=D\left(A_{p}\right) \cap L_{\sigma}^{p}(G) .
$$

Proof. We only have to show, that the right-hand side is a subset of $D\left(A_{S}\right)$. To this end, let $u \in$ $D\left(A_{p}\right) \cap L_{\sigma}^{p}(G)$ and $f:=A_{p} u$. It remains to show that $f \in L_{\sigma}^{p}(G)$. By the fact that $f=A_{p} u=$ curl $^{\prime} \operatorname{curl} u$ and $u \in D\left(A_{p}\right) \cap L_{\sigma}^{p}(G)$, the Gauß theorem yields

$$
\begin{aligned}
\int_{G} f \cdot \nabla \varphi d\left(x_{1}, x_{2}\right) & =\int_{G}\left(\operatorname{curl}^{\prime} \operatorname{curl} u\right) \cdot \nabla \varphi d\left(x_{1}, x_{2}\right) \\
& =-\left\langle\operatorname{curl} u, \nu \cdot \operatorname{curl}^{\prime} \varphi\right\rangle_{W_{p}^{1-1 / p}(\partial G), W_{p^{\prime}}^{-1 / p^{\prime}}(\partial G)}=0
\end{aligned}
$$

for all $\varphi \in \widehat{W}^{1, p^{\prime}}(G)$. Note that $\operatorname{div} \operatorname{curl}^{\prime} \varphi=0$, hence the trace $\nu \cdot \operatorname{curl}^{\prime} \varphi$ is defined in $W_{p^{\prime}}^{-1 / p^{\prime}}(\partial G)$ in the usual sense. By the fact that $\operatorname{curl} u \in W^{1, p}\left(G, \mathbb{R}^{2}\right)$ therefore the duality pairing on the boundary above is well-defined. The proof is complete.

Recall from (2.10) that $L^{p}\left(\Omega, \mathbb{R}^{2}\right)$ is decomposed in $L^{p}\left(\mathbb{R}, E_{3}^{p}\right)$ and $L^{p}\left(\mathbb{R},\left\langle e_{0}, e_{1}, e_{2}\right\rangle\right)$ with $E_{m}^{p}$ defined in the lines before (2.10) and $e_{0}, e_{1}, e_{2}$ the normed eigenfunctions to the first three eigenvalues of the operator $\mathcal{T}_{p, \theta}$ introduced in Subsection 2.3 .

In order to transfer the properties of $\mathbb{A}_{p}$ to the Stokes operator $A_{S}$ a crucial point is that $\widetilde{\Theta}_{*}^{p} L^{p}\left(\mathbb{R},\left\langle e_{0}, e_{1}, e_{2}\right\rangle\right)$ does not contain non-trivial solenoidal vector fields. To carry over full Sobolev regularity, however, this fact is not enough. This purpose requires stronger properties:

Proposition 3.2. Let $1<p<\infty$. Then there exists a $\delta>0$ such that

(1) $\|\widetilde{\mathbb{Q}} u\|_{p} \geq \delta\|u\|_{p}$ for all $u \in L_{\sigma}^{p}(G)$,

(2) $\|\mathbb{Q} u\|_{K_{p}^{2}} \geq \delta\|u\|_{K_{p}^{2}}$ for all $u \in D\left(B_{p}\right)$ such that $\operatorname{div} u=0$, and

(3) $\|\mathbb{Q} u\|_{D\left(A_{p}\right)} \geq \delta\|u\|_{D\left(A_{p}\right)}$ for all $u \in D\left(A_{S}\right)$.

Remark 3.3. Proposition 3.2 relies of course on the specific structure of the solenoidal subspace. In fact, its proof (including the proof of the subsequent Lemma 3.4) shows that the operator 'div' is isomorphic on the complemented space to $\mathbb{L}^{p}$ and on the corresponding higher order complemented subspaces. Furthermore, it keeps the complemented structure in its image. This essentially can be read off the representations of the transformed 'div' operator applied on elements of the complemented subspaces given in (3.5) and (3.10) below.

Proof of Lemma 3.2(1). Step 1. Recall from Subsection 2.3 that the eigenfunctions to the first three eigenvalues $\left(\lambda_{i}\right)_{i \in\{0,1,2\}} \in \sigma\left(\mathcal{T}_{p, \theta}\right)$ are explicitly given as

- $e_{0}(\theta):=\frac{1}{\sqrt{\theta_{0}}}\left(\begin{array}{l}1 \\ 0\end{array}\right)$ which corresponds to $\lambda_{0}=-1$ and

- $e_{k}(\theta):=\frac{1}{\sqrt{\theta_{0}}}\left(\begin{array}{c}\cos \left(\frac{k \pi}{\theta_{0}} \theta\right) \\ -\sin \left(\frac{k \pi}{\theta_{0}} \theta\right)\end{array}\right)$ which corresponds to $\lambda_{k}:=-\left(\frac{k \pi}{\theta_{0}}-1\right)^{2}$ for $k \in\{1,2\}$. 
We notice that, depending on the value of the angle $\theta_{0}$, there might be a doubled eigenvalue. This, however, does not matter for what follows. An element $\varphi \in L^{p}\left(\mathbb{R},\left\langle e_{0}, e_{1}, e_{2}\right\rangle\right)$ is then represented by

$$
\varphi(x, \theta)=\varphi_{0}(x) e_{0}(\theta)+\varphi_{1}(x) e_{1}(\theta)+\varphi_{2}(x) e_{2}(\theta)
$$

with coefficients $\varphi_{i} \in L^{p}(\mathbb{R})$ for $i \in\{0,1,2\}$.

Step 2. On our way to show (1) we first derive suitable estimates for $\varphi \in L^{p}\left(\mathbb{R},\left\langle e_{0}, e_{1}, e_{2}\right\rangle\right)$ in terms of the transformed divergence operator. To this end, first observe that

$$
\operatorname{div} \widetilde{\Theta}_{*}^{p} v \circ \psi=e^{\left(\beta_{p}-3\right) x}\left(\left(\beta_{p}-1+\partial_{x}\right) v_{x}+\partial_{\theta} v_{\theta}\right)=: e^{\left(\beta_{p}-3\right) x} \operatorname{div}_{\widetilde{\Theta}} v .
$$

Applying the transformed divergence operator $\operatorname{div}_{\widetilde{\Theta}}$ to representation (3.4) yields

$$
\begin{aligned}
\operatorname{div}_{\widetilde{\Theta}} \varphi= & \left(\beta_{p}-1+\partial_{x}\right) \varphi_{0} b_{0}+\left(\beta_{p}-1-\frac{\pi}{\theta_{0}}+\partial_{x}\right) \varphi_{1} b_{1} \\
& +\left(\beta_{p}-1-\frac{2 \pi}{\theta_{0}}+\partial_{x}\right) \varphi_{2} b_{2}
\end{aligned}
$$

where

$$
\left\{b_{0}, b_{1}, b_{2}\right\}:=\left\{\frac{1}{\sqrt{\theta_{0}}}, \frac{\cos \left(\frac{\pi}{\theta_{0}} \cdot\right)}{\sqrt{\theta_{0}}}, \frac{\cos \left(\frac{2 \pi}{\theta_{0}} \cdot\right)}{\sqrt{\theta_{0}}}\right\}
$$

is linearly independent in $L^{p}(I, \mathbb{R})$. We set

$$
F_{3}^{p}:=\left\langle b_{0}, b_{1}, b_{2}\right\rangle .
$$

The form of the coefficients in (3.5) is

$$
\left(s_{j}+\partial_{x}\right) \varphi_{j}, \quad s_{j} \in \mathbb{R}, j=0,1,2 .
$$

Observe that depending on the values of $p$ and $\theta_{0}$ it can occur $s_{j}=0$. Thus, in order to estimate expression (3.5) by $\varphi_{j}$ from below we distinguish two cases: $s_{j} \neq 0$ for all $j=0,1,2$ or $s_{j}=0$ for one $j \in\{0,1,2\}$.

Step 2.1. The case $s_{j} \neq 0$ for all $j=0,1,2$. Then we have

$$
s_{j}+\partial_{x} \in \mathscr{L}_{i s}\left(L^{p}(\mathbb{R}), W^{-1, p}(\mathbb{R})\right) .
$$

Furthermore, since $F_{3}^{p^{\prime}}$ is finite dimensional, we observe that $W^{1, p^{\prime}}\left(\mathbb{R}, F_{3}^{p^{\prime}}\right)$ is isomorphic to the space

$$
W^{1, p^{\prime}}\left(\mathbb{R}, F_{3}^{p^{\prime}}\right) \cap L^{p^{\prime}}\left(\mathbb{R}, W^{1, p^{\prime}}(I, \mathbb{R})\right) .
$$

This implies that the norm of $W^{1, p^{\prime}}\left(\mathbb{R}, F_{3}^{p^{\prime}}\right)$ and the norm of $W^{1, p^{\prime}}(\Omega, \mathbb{R})$ are equivalent on $W^{1, p^{\prime}}\left(\mathbb{R}, F_{3}^{p^{\prime}}\right)$ and that the latter space can be regarded as a closed subspace of $W^{1, p^{\prime}}(\Omega, \mathbb{R})$. Utilizing these facts, we can estimate as

$$
\begin{aligned}
\left\|\varphi_{j}\right\|_{p} & \leq C\left\|\left(s_{j}+\partial_{x}\right) \varphi_{j}\right\|_{W^{-1, p}(\mathbb{R})} \\
& \leq C\left\|\sum_{j=0}^{2}\left(s_{j}+\partial_{x}\right) \varphi_{j} b_{j}\right\|_{W^{-1, p}\left(\mathbb{R}, F_{3}^{p}\right)}=C\left\|\operatorname{div}_{\widetilde{\Theta}} \varphi\right\|_{W^{-1, p}\left(\mathbb{R}, F_{3}^{p}\right)} \\
& =C \sup _{0 \neq h \in W^{1, p^{\prime}}\left(\mathbb{R}, F_{3}^{p^{\prime}}\right)} \frac{\left|\left\langle h, \operatorname{div}_{\widetilde{\Theta}} \varphi\right\rangle\right|}{\|h\|_{W^{1, p^{\prime}}\left(\mathbb{R}, F_{3}^{p^{\prime}}\right)}}
\end{aligned}
$$




$$
\leq C \sup _{0 \neq h \in W^{1, p^{\prime}}(\Omega, \mathbb{R})} \frac{\left|\left\langle h, \operatorname{div}_{\widetilde{\Theta}} \varphi\right\rangle\right|}{\|h\|_{W^{1, p^{\prime}}(\Omega, \mathbb{R})}}=\left\|\operatorname{div}_{\widetilde{\Theta}} \varphi\right\|_{W_{0}^{-1, p}(\Omega, \mathbb{R})},
$$

for $j=0,1,2$ with $C>0$ independent of $\varphi$ and where $W_{0}^{-1, p}(\Omega, \mathbb{R})=\left(W^{1, p^{\prime}}(\Omega, \mathbb{R})\right)^{\prime}$.

Step 2.2. The case $s_{\ell}=0$ for one $\ell \in\{0,1,2\}$. This case is more involved, since here we have

$$
s_{\ell}+\partial_{x}=\partial_{x} \in \mathscr{L}_{i s}\left(L^{p}(\mathbb{R}), \widehat{W}^{-1, p}(\mathbb{R})\right),
$$

whereas for the remaining $j \in\{0,1,2\} \backslash\{\ell\}$ we still have (3.7). We set

$$
U_{j}:= \begin{cases}\widehat{W}^{-1, p}\left(\mathbb{R},\left\langle b_{j}\right\rangle\right), & \text { if } j=\ell, \\ W^{-1, p}\left(\mathbb{R},\left\langle b_{j}\right\rangle\right), & \text { if } j \in\{0,1,2\} \backslash\{\ell\}\end{cases}
$$

and

$$
V:={\overline{\operatorname{div}_{\widetilde{\Theta}} L^{p}\left(\mathbb{R}, E_{3}^{p}\right)}}^{W^{-1, p}(\Omega, \mathbb{R})} .
$$

In Lemma 3.4 below it is proved that the sum of $U_{0} \oplus U_{1} \oplus U_{2}$ and $V$ is direct and consequently that

$$
U_{0} \oplus U_{1} \oplus U_{2} \oplus V, \quad\|\cdot\|_{U_{0} \oplus U_{1} \oplus U_{2} \oplus V}:=\|\cdot\|_{U_{0}}+\|\cdot\|_{U_{1}}+\|\cdot\|_{U_{2}}+\|\cdot\|_{V}
$$

is a Banach space. Then, this time we obtain

$$
\begin{aligned}
\left\|\varphi_{j}\right\|_{p} & \leq C\left\|\left(s_{j}+\partial_{x}\right) \varphi_{j} b_{j}\right\|_{U_{j}} \\
& \leq C\left\|\sum_{j=0}^{2}\left(s_{j}+\partial_{x}\right) \varphi_{j} b_{j}\right\|_{U_{0} \oplus U_{1} \oplus U_{2}}=C\left\|\operatorname{div}_{\widetilde{\Theta}} \varphi\right\|_{U_{0} \oplus U_{1} \oplus U_{2}} \\
& \leq C\left\|\operatorname{div}_{\widetilde{\Theta}} \varphi\right\|_{U_{0} \oplus U_{1} \oplus U_{2} \oplus V}
\end{aligned}
$$

for $j=0,1,2$ with $C>0$ independent of $\varphi$.

Step 3. Now, let $u \in L_{\sigma}^{p}(G)$ and $\varphi \in L^{p}\left(\mathbb{R},\left\langle e_{0}, e_{1}, e_{2}\right\rangle\right)$ such that $\widetilde{\mathbb{Q}} u=u-\widetilde{\Theta}_{*}^{p} \varphi$. Observe that both,

and by Lemma 3.4 also

$$
\operatorname{div}_{\widetilde{\Theta}}: L^{p}\left(\Omega, \mathbb{R}^{2}\right) \rightarrow W_{0}^{-1, p}(\Omega, \mathbb{R})
$$

$$
\operatorname{div}_{\widetilde{\Theta}}: L^{p}\left(\Omega, \mathbb{R}^{2}\right) \rightarrow U_{0} \oplus U_{1} \oplus U_{2} \oplus V
$$

are bounded operators. By the fact that $\operatorname{div}_{\widetilde{\Theta}} \widetilde{\Theta}_{p}^{*} u=0$, we can continue the calculations in steps 2.1 and 2.2 to the result that

$$
\begin{aligned}
\left\|\varphi_{j}\right\|_{p} & \leq C\left\|\operatorname{div}_{\widetilde{\Theta}} \varphi\right\|_{\mathcal{W}}=C\left\|\operatorname{div}_{\widetilde{\Theta}}\left(\widetilde{\Theta}_{p}^{*} u-\varphi\right)\right\|_{\mathcal{W}} \\
& \leq C\left\|u-\widetilde{\Theta}_{*}^{p} \varphi\right\|_{L^{p}\left(G, \mathbb{R}^{2}\right)}=C\|\widetilde{\mathbb{Q}} u\|_{p} \quad(j=0,1,2),
\end{aligned}
$$

where $\mathcal{W}$ denotes either the space $W_{0}^{-1, p}(\Omega, \mathbb{R})$ or the space $U_{0} \oplus U_{1} \oplus U_{2} \oplus V$, depending on whether we have $s_{j} \neq 0$ for all $j$ or $s_{j}=0$ for one $j$. Summing up over $j$ yields

$$
\|\varphi\|_{p}=\|\varphi\|_{L^{p}\left(\mathbb{R},\left\langle e_{0}, e_{1}, e_{2}\right\rangle\right)} \leq C \sum_{j=0}^{2}\left\|\varphi_{j}\right\|_{p} \leq C\|\widetilde{\mathbb{Q}} u\|_{p}
$$

for all $u \in L_{\sigma}^{p}(G)$ and $\widetilde{\Theta}_{*}^{p} \varphi=(1-\widetilde{\mathbb{Q}}) u$. By the fact that

$$
C_{0}\|\varphi\|_{L^{p}\left(\Omega, \mathbb{R}^{2}\right)} \geq\left\|\widetilde{\Theta}_{*}^{p} \varphi\right\|_{L^{p}\left(G, \mathbb{R}^{2}\right)}=\|u-\widetilde{\mathbb{Q}} u\|_{p} \geq\|u\|_{p}-\|\widetilde{\mathbb{Q}} u\|_{p}
$$

we arrive at (1) by setting $\delta:=1 /\left(C_{0} C+1\right)$. 
Proof of (2). The proof of (2) is in large parts similar to the proof of (1). Hence we will be briefer in detail.

Step 1. Again we will first provide estimates for $\varphi \in\left(1-\mathbb{P}_{3}\right) \Theta_{p}^{*} D\left(B_{p}\right)$ in terms of the transformed divergence. Note that such a $\varphi$ is still represented by (3.4), but now with coefficients $\varphi_{j} \in W^{2, p}(\mathbb{R})$. The transformed divergence operator here is

$$
\operatorname{div} \Theta_{*}^{p} v \circ \psi=e^{\left(\beta_{p}-1\right) x}\left(\left(\beta_{p}+1+\partial_{x}\right) v_{x}+\partial_{\theta} v_{\theta}\right)=: e^{\left(\beta_{p}-1\right) x} \operatorname{div}_{\Theta} v .
$$

Consequently,

$$
\begin{aligned}
\operatorname{div}_{\Theta} \varphi & =\left(\beta_{p}+1+\partial_{x}\right) \varphi_{0} b_{0}+\left(\beta_{p}+1-\frac{\pi}{\theta_{0}}+\partial_{x}\right) \varphi_{1} b_{1} \\
& +\left(\beta_{p}+1-\frac{2 \pi}{\theta_{0}}+\partial_{x}\right) \varphi_{2} b_{2}
\end{aligned}
$$

for $\varphi \in\left(1-\mathbb{P}_{3}\right) \Theta_{p}^{*} D\left(B_{p}\right)$. Again we write the coefficients as $\left(s_{j}+\partial_{x}\right) \varphi_{j}$. Here still $s_{1}$ and $s_{2}$ can vanish. Hence we again distinguish the two cases: $s_{j} \neq 0$ for all $j=0,1,2$ or $s_{j}=0$ for one $j \in\{1,2\}$.

Step 1.1. For the case $s_{j} \neq 0$ for all $j=0,1,2$ we use

$$
s_{j}+\partial_{x} \in \mathscr{L}_{i s}\left(W^{2, p}(\mathbb{R}), W^{1, p}(\mathbb{R})\right)
$$

in order to deduce

$$
\begin{aligned}
\left\|\varphi_{j}\right\|_{W^{2, p}(\mathbb{R})} & \leq C\left\|\left(s_{j}+\partial_{x}\right) \varphi_{j}\right\|_{W^{1, p}(\mathbb{R})} \\
& \leq C\left\|\sum_{j=0}^{2}\left(s_{j}+\partial_{x}\right) \varphi_{j} b_{j}\right\|_{W^{1, p}\left(\mathbb{R}, F_{3}^{p}\right)} \leq C\left\|\operatorname{div}_{\Theta} \varphi\right\|_{W^{1, p}(\Omega, \mathbb{R})}
\end{aligned}
$$

for $j=0,1,2$ with $C>0$ independent of $\varphi$.

Step 1.2. If $s_{\ell}=0$ for one $\ell \in\{1,2\}$ we use for that $\ell$,

$$
s_{\ell}+\partial_{x}=\partial_{x} \in \mathscr{L}_{i s}\left(\widehat{W}^{2, p}(\mathbb{R}), \widehat{W}^{1, p}(\mathbb{R})\right)
$$

to estimate

$$
\begin{aligned}
\left\|\varphi_{\ell}\right\|_{W^{2, p}(\mathbb{R})} & \leq C\left\|\left(s_{\ell}+\partial_{x}\right) \varphi_{\ell}\right\|_{\widehat{W}^{1, p}(\mathbb{R})} \leq C\left\|\left(s_{\ell}+\partial_{x}\right) \varphi_{\ell}\right\|_{W^{1, p}(\mathbb{R})} \\
& \leq C\left\|\sum_{j=0}^{2}\left(s_{j}+\partial_{x}\right) \varphi_{j} b_{j}\right\|_{W^{1, p}\left(\mathbb{R}, F_{3}^{p}\right)} \leq C\left\|\operatorname{div}_{\Theta} \varphi\right\|_{W^{1, p}(\Omega, \mathbb{R})}
\end{aligned}
$$

with $C>0$ independent of $\varphi$. The corresponding estimate for $\varphi$ in the $L^{p}$-norm can be established completely analogous as in step 2.2 of the proof of (1). In this regard, observe that all assertions there as well as of Lemma 3.4 obviously remain true, if we replace $\operatorname{div}_{\widetilde{\Theta}}$ by $\operatorname{div}_{\Theta}$. Hence we obtain

$$
\left\|\varphi_{\ell}\right\|_{L^{p}(\mathbb{R})} \leq C\left\|\operatorname{div}_{\Theta} \varphi\right\|_{U_{0} \oplus U_{1} \oplus U_{2} \oplus V} .
$$

Taking into account the well-known interpolation estimate $\|\nabla v\|_{L^{p}(\mathbb{R})} \leq C\left(\left\|\nabla^{2} v\right\|_{L^{p}(\mathbb{R})}+\|v\|_{L^{p}(\mathbb{R})}\right)$, altogether we have

$$
\left\|\varphi_{j}\right\|_{W^{2, p}(\mathbb{R})} \leq C\left(\left\|\operatorname{div}_{\Theta} \varphi\right\|_{W^{1, p}(\Omega, \mathbb{R})}+\left\|\operatorname{div}_{\Theta} \varphi\right\|_{U_{0} \oplus U_{1} \oplus U_{2} \oplus V}\right)
$$

for $j=0,1,2$ with $C>0$ independent of $\varphi$. 
Step 2. Let $u \in D\left(B_{p}\right)$ with $\operatorname{div} u=0$ and $\varphi \in\left(1-\mathbb{P}_{3}\right) \Theta_{p}^{*} D\left(B_{p}\right)$ such that $\mathbb{Q} u=u-\Theta_{*}^{p} \varphi$. Thanks to Lemma 3.4 and since

$$
\operatorname{div}_{\Theta}: W^{2, p}\left(\Omega, \mathbb{R}^{2}\right) \rightarrow W^{1, p}(\Omega, \mathbb{R})
$$

is bounded, by virtue of $\operatorname{div}_{\Theta} \Theta_{p}^{*} u=0$ and the estimates in Steps 1.1 and 1.2 we conclude

$$
\begin{aligned}
\left\|\varphi_{j}\right\|_{W^{2, p}(\mathbb{R})} & \leq C\left(\left\|\operatorname{div}_{\Theta} \varphi\right\|_{W^{1, p}(\Omega, \mathbb{R})}+\left\|\operatorname{div}_{\Theta} \varphi\right\|_{U_{0} \oplus U_{1} \oplus U_{2} \oplus V}\right) \\
& \leq C\left\|\Theta_{p}^{*} u-\varphi\right\|_{W^{2, p}(\Omega, \mathbb{R})} \\
& \leq C\left\|u-\Theta_{*}^{p} \varphi\right\|_{K_{p}^{2}\left(G, \mathbb{R}^{2}\right)}=C\|\mathbb{Q} u\|_{K_{p}^{2}\left(G, \mathbb{R}^{2}\right)} \quad(j=0,1,2) .
\end{aligned}
$$

Summing up over $j$, analogous to step 3 of the proof of (1) we arrive at (2).

Proof of (3). According to Lemma 2.17(1), $\|\cdot\|_{p}+\|\cdot\|_{K_{p}^{2}}$ is an equivalent norm on $D\left(A_{p}\right)$ and we have $\mathbb{Q}=\widetilde{\mathbb{Q}}$ on $D\left(A_{p}\right)$. The estimates proved in (1) and (2) then yield

$$
\begin{aligned}
\|u\|_{D\left(A_{p}\right)} & \leq C\left(\|u\|_{p}+\|u\|_{K_{p}^{2}}\right) \leq C\left(\|\mathbb{Q} u\|_{p}+\|\mathbb{Q} u\|_{K_{p}^{2}}\right) \\
& \leq C\|\mathbb{Q} u\|_{D\left(A_{p}\right)} \quad\left(u \in D\left(A_{S}\right)\right) .
\end{aligned}
$$

The proof is now completed.

We have used the following facts in the proof of Proposition 3.2 .

Lemma 3.4. Let $1<p<\infty$. Let $U_{j}, j=0,1,2, \operatorname{div}_{\widetilde{\Theta}}$, and $V$ be as defined in the proof of Proposition 3.2(1). Then $U_{0}, U_{1}, U_{2}, V$ are Banach spaces, their sum is direct, and we have

$$
\operatorname{div}_{\widetilde{\Theta}} \in \mathscr{L}\left(L^{p}\left(\Omega, \mathbb{R}^{2}\right), U_{0} \oplus U_{1} \oplus U_{2} \oplus V\right) .
$$

Proof. By their definition (3.8) and (3.9) it is obvious that $U_{0}, U_{1}, U_{2}, V$ are Banach spaces and that the sum of $U_{0}, U_{1}, U_{2}$ is direct. Note that

$$
L^{p}\left(\Omega, \mathbb{R}^{2}\right)=L^{p}\left(\mathbb{R}, E_{3}^{p}\right) \oplus L^{p}\left(\mathbb{R},\left\langle e_{0}\right\rangle\right) \oplus L^{p}\left(\mathbb{R},\left\langle e_{1}\right\rangle\right) \oplus L^{p}\left(\mathbb{R},\left\langle e_{2}\right\rangle\right) .
$$

It is also obvious that $\operatorname{div}_{\widetilde{\Theta}}: L^{p}\left(\mathbb{R},\left\langle e_{j}\right\rangle\right) \rightarrow U_{j}$ and hence also

$$
\operatorname{div}_{\widetilde{\Theta}}: L^{p}\left(\mathbb{R},\left\langle e_{0}, e_{1}, e_{2}\right\rangle\right) \rightarrow U_{0} \oplus U_{1} \oplus U_{2}
$$

is bounded (even isomorphic due to the estimates for $\varphi$ in steps 2.1 and 2.2 of the proof of Proposition $[3.2)$. Due to $\operatorname{div}_{\widetilde{\Theta}} \in \mathscr{L}\left(L^{p}\left(\Omega, \mathbb{R}^{2}\right), W^{-1, p}(\Omega, \mathbb{R})\right)$ we see that by definition of $V$ the operator

$$
\operatorname{div}_{\widetilde{\Theta}}: L^{p}\left(\mathbb{R}, E_{3}^{p}\right) \rightarrow V
$$

is bounded too. It remains to prove that the sum of $V$ and $U_{0} \oplus U_{1} \oplus U_{2}$ is direct.

To this end, denote by $\mathcal{Q}_{3}: W^{1, p^{\prime}}(\Omega, \mathbb{R}) \rightarrow W^{1, p^{\prime}}(\Omega, \mathbb{R})$ the projector

$$
\mathcal{Q}_{3} v:=\sum_{j=0}^{2}\left(v, b_{j}\right) b_{j}, \quad v \in W^{1, p^{\prime}}(\Omega, \mathbb{R})
$$

with $b_{j}, j=0,1,2$, be defined as in (3.6). Writing

$$
W^{1, p^{\prime}}(\Omega, \mathbb{R})=W^{1, p^{\prime}}\left(\mathbb{R}, L^{p^{\prime}}(I, \mathbb{R})\right) \cap L^{p^{\prime}}\left(\mathbb{R}, W^{1, p^{\prime}}(I, \mathbb{R})\right)
$$

it is easily seen that $\mathcal{Q}_{3}$ is a bounded projector onto $W^{1, p^{\prime}}\left(\mathbb{R}, F_{3}^{p^{\prime}}\right)$. Note that $\left(b_{k}\right)_{k=0}^{\infty}$ with $b_{k}(\theta)=$ $\cos \left(k \pi \theta / \theta_{0}\right) / \sqrt{\theta_{0}}$ as the collection of eigenfunctions of the Neumann-Laplacian on the interval $I=$ 
$\left(0, \theta_{0}\right)$ forms an orthonormal Hilbert basis of $L^{2}(I, \mathbb{R})$. This shows that $\mathcal{Q}_{3}$ is symmetric, hence $\mathcal{Q}_{3}$ is a bounded projector on

$$
W_{0}^{-1, p}(\Omega, \mathbb{R})=\left(W_{0}^{1, p^{\prime}}(\Omega, \mathbb{R})\right)^{\prime}=W^{-1, p}\left(\mathbb{R}, L^{p}(I, \mathbb{R})\right)+L^{p}\left(\mathbb{R}, W_{0}^{-1, p}(I, \mathbb{R})\right),
$$

too. Since all norms on $F_{3}^{p}$ are equivalent, for its image we calculate

$$
\begin{aligned}
\mathcal{Q}_{3} W_{0}^{-1, p}(\Omega, \mathbb{R}) & =W^{-1, p}\left(\mathbb{R}, F_{3}^{p}\right)+L^{p}\left(\mathbb{R}, F_{3}^{p}\right)=W^{-1, p}\left(\mathbb{R}, F_{3}^{p}\right) \\
& =W^{-1, p}\left(\mathbb{R},\left\langle b_{0}\right\rangle\right) \oplus W^{-1, p}\left(\mathbb{R},\left\langle b_{1}\right\rangle\right) \oplus W^{-1, p}\left(\mathbb{R},\left\langle b_{2}\right\rangle\right) .
\end{aligned}
$$

We next show that $V \subset\left(1-\mathcal{Q}_{3}\right) W_{0}^{-1, p}(\Omega, \mathbb{R})$. By the fact that $\left(e_{k}\right)_{k=0}^{\infty}$ forms a basis of $L^{2}\left(I, \mathbb{R}^{2}\right)$ (see (2.6) and the subsequent lines), every $v \in L^{2}\left(\mathbb{R}, E_{3}^{2}\right)$ is represented as $v=\sum_{k=3}^{\infty} v_{k} e_{k}$ with $\left(v_{k}\right) \subset L^{2}(\mathbb{R})$. Hence we obtain

$$
\begin{aligned}
\operatorname{div}_{\widetilde{\Theta}} v & =\sum_{k=3}^{\infty}\left(\beta_{2}-1+\partial_{x}\right) v_{k} e_{k}^{1}+v_{k} \partial_{\theta} e_{k}^{2} \\
& =\sum_{k=3}^{\infty}\left(\beta_{2}-1 \pm \frac{k \pi}{\theta_{0}}+\partial_{x}\right) v_{k} b_{k}
\end{aligned}
$$

This shows that

$$
\mathcal{Q}_{3} \operatorname{div}_{\widetilde{\Theta}} v=0 \quad\left(v \in L^{p}\left(\mathbb{R}, E_{3}^{p}\right) \cap L^{2}\left(\mathbb{R}, E_{3}^{2}\right)\right) .
$$

The boundedness of the operators $\operatorname{div}_{\widetilde{\Theta}}, \mathcal{Q}_{3}$ and a density argument yield that this identity remains true for all $v \in L^{p}\left(\mathbb{R}, E_{3}^{p}\right)$. Once more the boundedness of $\mathcal{Q}_{3}$ on $W_{0}^{-1, p}(\Omega, \mathbb{R})$ then gives $V \subset$ $\left(1-\mathcal{Q}_{3}\right) W_{0}^{-1, p}(\Omega, \mathbb{R})$.

Finally, $W^{1, p}\left(\mathbb{R},\left\langle b_{j}\right\rangle\right) \stackrel{d}{\hookrightarrow} \widehat{W}^{1, p}\left(\mathbb{R},\left\langle b_{j}\right\rangle\right)$ implies

$$
\widehat{W}^{-1, p}\left(\mathbb{R},\left\langle b_{j}\right\rangle\right) \hookrightarrow W^{-1, p}\left(\mathbb{R},\left\langle b_{j}\right\rangle\right) .
$$

In combination with (3.14) this gives

$$
U_{0} \oplus U_{1} \oplus U_{2} \subset \mathcal{Q}_{3} W_{0}^{-1, p}(\Omega, \mathbb{R}),
$$

hence $V \cap\left(U_{0} \oplus U_{1} \oplus U_{2}\right)=\{0\}$.

Since we equip $U_{0} \oplus U_{1} \oplus U_{2} \oplus V$ with the norm $\|\cdot\|_{U_{0} \oplus U_{1} \oplus U_{2} \oplus V}:=\|\cdot\|_{U_{0}}+\|\cdot\|_{U_{1}}+\|\cdot\|_{U_{2}}+\|\cdot\|_{V}$, relations (3.12) and (3.13) result in (3.11). Now all assertions are proved.

Corollary 3.5. Let $1<p<\infty$. Then we have that

(1) $\widetilde{\mathbb{Q}} L_{\sigma}^{p}(G)$ is closed in $\mathbb{L}^{p}$ and $\widetilde{\mathbb{Q}} \in \mathscr{L}_{i s}\left(L_{\sigma}^{p}(G), \widetilde{\mathbb{Q}} L_{\sigma}^{p}(G)\right)$,

(2) $\mathbb{Q} D_{\sigma}$ is closed in $D\left(\mathbb{B}_{p}\right)$ and $\mathbb{Q} \in \mathscr{L}_{i s}\left(D_{\sigma}, \mathbb{Q} D_{\sigma}\right)$, where $D_{\sigma}:=\left\{v \in D\left(\mathbb{B}_{p}\right): \operatorname{div} v=0\right\}$, and

(3) $\mathbb{Q} D\left(A_{S}\right)$ is closed in $D\left(\mathbb{A}_{p}\right)$ and $\mathbb{Q} \in \mathscr{L}_{\text {is }}\left(D\left(A_{S}\right), \mathbb{Q} D\left(A_{S}\right)\right)$.

With these facts at hand we can prove our main result on the Stokes operator.

Proof of Theorem 1.2. Assume that $\lambda \in \rho\left(A_{p}\right)$. By the fact that $A_{S}$ is the part of $A_{p}$ from Lemma 3.1 we infer that

$$
\left(\lambda-A_{S}\right)^{-1}=\left.\left(\lambda-A_{p}\right)^{-1}\right|_{L_{\sigma}^{p}(G)} .
$$

In combination with Lemma 2.17(3),(4) this implies

$$
\mathbb{Q}\left(\lambda-A_{S}\right)^{-1} u=\left(\lambda-\mathbb{A}_{p}\right)^{-1} \widetilde{\mathbb{Q}} u \quad\left(u \in D\left(A_{S}\right)\right) .
$$


In particular, the above line yields $\left(\lambda-\mathbb{A}_{p}\right)^{-1} \widetilde{\mathbb{Q}} L_{\sigma}^{p}(G) \subset \mathbb{Q} D\left(A_{S}\right)$. Thus, thanks to Corollary 3.5 we conclude that

$$
\left(\lambda-A_{S}\right)^{-1} f=\mathbb{Q}^{-1}\left(\lambda-\mathbb{A}_{p}\right)^{-1} \widetilde{\mathbb{Q}} f \quad\left(L_{\sigma}^{p}(G)\right) .
$$

For $1<p<1+\delta$ with $\delta>0$ given in Theorem 2.13 we know by that result that the resolvent set of $A_{p}$ contains a suitable sector. For those $p$ the assertion hence follows from Corollary 3.5 and Theorem 2.23. For general $p \in(1, \infty)$ representation (3.15) gives a candidate for the resolvent of $A_{S}$. In fact, choosing $1<q<1+\delta$, on $L_{\sigma}^{p}(G) \cap L_{\sigma}^{q}(G)$ we already know that it is the resolvent. A density argument and again Corollary 3.5 and Theorem 2.23 then yield the assertion.

Remark 3.6. From Proposition 2.9(1) and Theorem 2.22 it also follows consistency of the resolvent of $A_{S}$, that is, for every $\lambda \in \rho\left(A_{S}\right)$ the family $\left(\left(\lambda-A_{S}\right)^{-1}\right)_{1<p<\infty}$ is consistent on the scale $\left(L_{\sigma}^{p}(G)\right)_{1<p<\infty}$.

Finally we prove our third main result.

Proof of Theorem 1.6. We follow the strategy in the proof of Theorem 1.2. For $f \in L_{\sigma}^{p}(G)$ the candidate for the solution of

$$
\left.\begin{array}{rll}
-\Delta u+\nabla \pi=f & \text { in } & G, \\
\operatorname{div} u=0 & \text { in } & G, \\
\operatorname{curl} u=0, u \cdot \nu=0 & \text { on } & \partial G
\end{array}\right\}
$$

is given as $\pi=0$ and $u=\mathbb{Q}^{-1} \mathbb{B}_{p}^{-1} \widetilde{\mathbb{Q}} f$. Thanks to Proposition 2.9 and Corollary 3.5 it remains to show that $\operatorname{div} u=0$. This, in turn, follows from Proposition $2.24, \mathbb{Q}^{-1}\left(\lambda-\mathbb{A}_{p}\right)^{-1} \widetilde{\mathbb{Q}} f \subset D\left(A_{S}\right)$, and the fact that the operator div acts continuously on the space $K_{p}^{2}\left(G, \mathbb{R}^{2}\right)$.

\section{Appendix A. Elements from harmonic And FUnCTIONAl ANALYsis}

The following facts might be well-known. Since we could not find an appropriate reference, we give their proofs here.

Lemma A.1. Let $X, Y$ be Banach spaces such that $X \hookrightarrow Y$. Then we have

$$
C_{c}^{\infty}(\mathbb{R}, X) \stackrel{d}{\hookrightarrow} W^{k, p}(\mathbb{R}, X) \cap W^{\ell, p}(\mathbb{R}, Y)
$$

for every $k, \ell \in \mathbb{N}_{0}$ and $p \in(1, \infty)$.

Proof. First recall that

$$
C_{c}^{\infty}(\mathbb{R}, E) \stackrel{d}{\hookrightarrow} W^{k, p}(\mathbb{R}, E)
$$

for every $k \in \mathbb{N}_{0}, p \in(1, \infty)$, and arbitrary Banach space $E$. In fact, it is standard to construct a (universal) sequence of operators $\left(\Phi_{k}\right)_{k \in \mathbb{N}}$ such that for $u \in W^{k, p}(\mathbb{R}, E)$ we have $\left(\Phi_{k} u\right) \subset C_{c}^{\infty}(\mathbb{R}, E)$ and

$$
\Phi_{k} u \rightarrow u \quad \text { in } W^{k, p}(\mathbb{R}, E) \quad(k \rightarrow \infty)
$$

for every $k \in \mathbb{N}_{0}, p \in(1, \infty)$, and arbitrary Banach space $E$. Since $X \subset Y$, for $u \in W^{k, p}(\mathbb{R}, X) \cap$ $W^{\ell, p}(\mathbb{R}, Y)$ this gives $\Phi_{k} u \rightarrow u$ in $W^{k, p}(\mathbb{R}, X)$ and in $W^{\ell, p}(\mathbb{R}, Y)$. 
Let $T: D(T) \subset X \rightarrow X$ be a closed, densely defined operator on a Banach space $X$. We denote by

$$
T^{\sharp}: X^{\prime} \rightarrow D(T)^{\prime}
$$

the dual operator of $T$, regarded as a bounded operator from $D(T)$ to $X$, and by

$$
T^{\prime}: D\left(T^{\prime}\right) \subset X^{\prime} \rightarrow X^{\prime}
$$

the usual Banach space dual operator of $T$. The fact that $D(T) \subset X$ is dense, obviously implies $D\left(T^{\prime}\right) \hookrightarrow X^{\prime} \hookrightarrow D(T)^{\prime}$ and that

$$
\left.T^{\sharp}\right|_{D\left(T^{\prime}\right)}=T^{\prime} .
$$

Furthermore, we have the following lemma on consistency.

Lemma A.2. Let $X$ be a reflexive Banach space and let $T: D(T) \subset X \rightarrow X$ be densely defined such that $T \in \mathscr{L}_{i s}(D(T), X)$. Assume there is an embedding (with means i.p. injection) $J: D(T) \rightarrow X^{\prime}$ with dense range. Then there exists an embedding $\widetilde{J}: X \rightarrow D(T)^{\prime}$ such that, if $\widetilde{J} \circ T \subset T^{\sharp} \circ J$, we have

$$
\left.J \circ T^{-1} \circ \widetilde{J}^{-1}\right|_{\widetilde{J} X \cap X^{\prime}}=\left.\left(T^{\sharp}\right)^{-1}\right|_{\widetilde{J} X \cap X^{\prime}}=\left.\left(T^{\prime}\right)^{-1}\right|_{\widetilde{J} X \cap X^{\prime}} \quad \text { in } X^{\prime} .
$$

Proof. Since $\overline{D(T)}=X$ we have $X^{\prime} \hookrightarrow D(T)^{\prime}$. Reflexivity of $X$ and $J(D(T)) \stackrel{d}{\hookrightarrow} X^{\prime}$ further imply that there is an embedding $\widetilde{J}: X \rightarrow D(T)^{\prime}$. Thus, $\widetilde{J} X \cap X^{\prime}$ is well-defined and due to $T \in \mathscr{L}_{i s}(D(T), X)$ which also implies $T^{\sharp} \in \mathscr{L}_{i s}\left(X^{\prime}, D(T)^{\prime}\right)$ and $T^{\prime} \in \mathscr{L}_{i s}\left(D\left(T^{\prime}\right), X^{\prime}\right)$, line (A.2) is meaningful.

Now, let $z \in \widetilde{J} X \cap X^{\prime}$ and set $x_{1}:=J T^{-1} \widetilde{J}^{-1} z \in X^{\prime}$ and $x_{2}:=\left(T^{\sharp}\right)^{-1} z \in X^{\prime}$. Thanks to $\widetilde{J} \circ T \subset T^{\sharp} \circ J$ we obtain

$$
\begin{aligned}
T^{\sharp}\left(x_{1}-x_{2}\right) & =T^{\sharp}\left(J T^{-1} \widetilde{J}^{-1} z-\left(T^{\sharp}\right)^{-1} z\right) \\
& =\widetilde{J} T T^{-1} \widetilde{J}^{-1} z-T^{\sharp}\left(T^{\sharp}\right)^{-1} z=z-z=0 .
\end{aligned}
$$

Thus $x_{1}=x_{2}$ in $X^{\prime}$ and the assertion is proved. The second equality in (A.2) follows in a similar manner from (A.1).

\section{REFERENCES}

[1] R.A. Adams and J. J. F. Fournier. Sobolev Spaces. Academic Press, 1975.

[2] S.-K. Chua. Extension theorems on weighted Sobolev spaces. Indiana Univ. Math. J., 41:1027-1076, 1992.

[3] C. De Coster, S. Nicaise: Singular behavior of the solution of the Helmholtz equation in weighted $L^{p}$-Sobolev spaces. Advances Diff. Eq. 16 (2011), 165-198.

[4] C. De Coster, S. Nicaise: Singular behavior of the solution of the Cauchy-Dirichlet heat equation in weighted $L^{p}$-Sobolev spaces. Bull. B.M.S.-Simon Stevin 11 (2011), 769-780.

[5] M. Dauge. Stationary Stokes and Navier-Stokes systems on two- or three-dimensional domains with corners. I. Linearized equations. SIAM J. Math. Anal., 20(1):74-97, 1989.

[6] R. Denk, M. Hieber, and J. Prüß. R-Boundedness, Fourier-Multipliers and Problems of Elliptic and Parabolic Type, volume 166 of Mem. Amer. Math. Soc. American Mathematical Society, 2003.

[7] P. Deuring. $L^{p}$-theory for the Stokes system in 3D domains with conical boundary points. Indiana Univ. Math. J., 47(1):11-47, 1998.

[8] P. Deuring. The Stokes resolvent in 3D domains with conical boundary points:non-regularity in $L^{p}$-spaces. $A d v$. Diff. Eq., 6:175-226, 2001.

[9] G.P. Galdi. An Introduction to the Mathematical Theory of the Navier-Stokes Equations. Springer, 2011.

[10] P. Grisvard. Elliptic Problems in Nonsmooth Domains. Society for Industrial and Applied Mathematics, 2011.

[11] B. Guo and C. Schwab. Analytic regularity of Stokes flow on polygonal domains in countably weighted Sobolev spaces. J. Comput. Appl. Math., 190(1-2):487-519, 2006. 
[12] M. Haase. The Functional Calculus for Sectorial Operators. Operator Theory Advances and Applications. Springer, Basel, 2006.

[13] M. Hieber and J. Saal. The Stokes Equation in the $L^{p}$-setting: Well Posedness and Regularity Properties, pages 1-88. Handbook of Mathematical Analysis in Mechanics of Viscous Fluids. Springer, 2016.

[14] P.W. Jones. Quasiconformal mappings and extendability of functions in Sobolev spaces. Acta Math., 147:71-88, 1981.

[15] M. Kaip and J. Saal. The Permanence of $\mathcal{R}$-Boundedness under Interpolation and Applications to Parabolic Systems. J. Math. Sci. Univ. Tokyo, 19:1-49, 2012.

[16] N. J. Kalton and L. Weis. The $H^{\infty}$-Calculus and Sums of Closed Operators. Math. Ann., 321:319-345, 2001.

[17] R.B. Kellogg and J.E. Osborn. A regularity result for the Stokes problem in a convex polygon. J. Funct. Anal., 21:397-431, 1976.

[18] V.A. Kondrat'ev. Boundary problems for elliptic equations in domains with conical or angular points. Trans. Mosc. Math. Soc, 16:227-313, 1967.

[19] V. Kozlov, J. Rossmann: On the nonstationary Stokes system in a cone. J. Diff. Equ. 260 (2016), 8277-8315.

[20] P. Kunstmann and L. Weis. Maximal $L_{p}$-Regularity for Parabolic Equations, Fourier Multiplier Theorems and $H^{\infty}$ Functional Calculus. In Functional Analytic Methods for Evolution Equations. volume 1855 of Lecture Notes in Mathematics, pages 65-311. Springer Berlin Heidelberg, 2004.

[21] S. Maier and J. Saal. Stokes and Navier Stokes Equations with Perfect Slip on Wedge Type Domains. Discrete Contin. Dyn. Syst.- Series S, 7(5):1045-1063, 2014.

[22] V.G. Maz'ja and J. Rossmann. Elliptic Equations in Polyhedral Domains. AMS, Providence, Rhode Island, 2010.

[23] M. Mitrea and S. Monniaux. The regularity of the Stokes operator and the Fujita-Kato approach to the Navier-Stokes initial value problem in Lipschitz domains. J. Funct. Anal., 254(6):1522-1574, 2008.

[24] T. Nau and J. Saal. $H^{\infty}$-Calculus for Cylindrical Boundary Value Problems. Adv. Differ. Equ., 17(7-8):767-800, 2012.

[25] A.I. Nazarov. $L_{p}$-estimates for a solution to the Dirichlet problem and to the Neumann problem for the heat equation in a wedge with edge of arbitrary codimension. J. Math. Sci., 106:2989-3014, 2001.

[26] J. Prüss and G. Simonett. $\mathrm{H}^{\infty}$-Calculus for the Sum of Non-Commuting Operators. Trans. Amer. Math. Soc, 359:3549-3565, 2007.

[27] J. Prüss and G. Simonett. Moving Interface and Quasilinear Parabolic Evolution Equations Monographs in Mathematics 105, Birkhäuser, (2016).

[28] Z. Shen. Resolvent estimates in $L_{p}$ for the Stokes operator in Lipschitz domains. Arch. Ration. Mech. Anal., 205(2):395-424, 2012.

[29] E.M. Stein. Singular Integrals and Differentiability Properties of Functions. Princeton University Press, 1970.

[30] P. Tolksdorf. On the $L^{p}$-theory of the Navier-Stokes equations on three-dimensional bounded lipschitz domains. arXiv:1703.01091.

[31] H. Triebel. Interpolation Theory, Function Spaces, Differential Operators. North Holland, 1978.

[32] J. Voigt. Abstract Stein interpolation. Math. Nachr., 157:197-199, 1992.

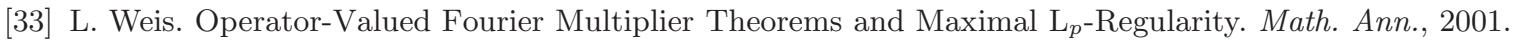

Mathematisches Institut, Angewandte Analysis, Heinrich-Heine-Universität Düsseldorf, 40204 Düsseldorf, Germany

E-mail address: matthias.koehne@hhu.de

E-mail address: juergen.saal@hhu.de

E-mail address: laura.westermann@hhu.de 